\title{
Grain boundary diffusion of chromium in polycrystalline nickel studied by SIMS
}

\author{
Thomas Gheno $^{\mathrm{a} *}$ (D), François Jomard ${ }^{\mathrm{b}}$, Clara Desgranges ${ }^{\mathrm{a}, \mathrm{c}}$ (ID and \\ Laure Martinelli ${ }^{\mathrm{a}}$ (ID \\ ${ }^{a}$ DEN-SCCME, CEA, Université Paris-Saclay, 91191 Gif-sur-Yvette, France \\ ${ }^{b}$ GEMaC, UMR 8635, Université de Versailles-Saint-Quentin, \\ 45 avenue des Etats-Unis, 78035 Versailles, France \\ ${ }^{c}$ Safran-Tech, rue des jeunes bois, Châteaufort, 78772 Magny-les-Hameaux, France
}

This is the accepted version of an article published in Materialia, available at: https://doi.org/10.1016/j.mtla.2019.100283

This version is made available under the CC-BY-NC-ND 4.0 license

\begin{abstract}
The grain boundary diffusion of chromium in polycrystalline nickel was studied by means of tracer experiments at $346-668{ }^{\circ} \mathrm{C}$. Intensity-depth profiles were recorded by secondary ion mass spectrometry (SIMS), which allowed short diffusion distances, and therefore relatively low temperatures, to be examined. Individual grain boundaries in coarse-grained substrates produced profiles with a variety of shapes, reflecting the variability of diffusivities in a polycrystal, and even within a grain boundary. Average diffusivities were also measured using cold-rolled substrates, which rapidly recrystallized and provided finer microstructures. The simplifying assumptions usually made in processing $\mathrm{B}$ regime data were found not to be applicable here, because of the shallow measurement depths. The errors made by using approximate solutions to the diffusion equation are discussed, and a data processing method adapted to the conditions of SIMS measurements is used. A method to study diffusion tails with smooth slope variations is also demonstrated. The measured diffusivities were found to be in agreement with higher temperature data from the literature.
\end{abstract}

Keywords: Tracer diffusion; Intergranular diffusion; Nickel alloys

\section{Introduction}

Short-circuit (phase boundary, grain boundary and dislocation) diffusion plays an important role in a number of processes underlying the elaboration and degradation of structural alloys, e.g., recrystallization, creep or stress corrosion cracking. Along with volume diffusion and thermodynamic data, short-circuit diffusion data are needed to gain a better understanding of microstructure transformations and to generate more accurate simulations of these transformations.

Activation energies for short-circuit diffusion are typically lower than those of volume diffusion [1]. As a consequence, the relative contribution of short-circuits to mass transport becomes more important at lower temperatures. The case of the Ni-based, Cr-bearing alloys used in high pressure water in nuclear power plants offers an illustration of the importance of short-circuits: in typical conditions of autoclave tests $\left(10^{2}-10^{3} \mathrm{~h}\right.$ at $\left.300-350^{\circ} \mathrm{C}\right)$, the volume diffusion distance of $\mathrm{Cr}$ in $\mathrm{Ni}$ is a fraction of a nanometer, and yet $\mathrm{Cr}$ depletion (due to the selective removal of

\footnotetext{
${ }^{*}$ Corresponding author. E-mail address: thomas.gheno@onera.fr
} 
Cr to form Cr-rich oxide) is occasionally detected over tens of nanometers from the alloy surface $[2,3]$ or from oxidized grain boundaries and intergranular cracks [4-6]. Intergranular oxidation is thought to be a predominant source of failure in these systems [7], which further warrants the need for a better understanding of shortcircuit diffusion, and for the acquisition of the associated kinetics data.

Grain boundary diffusion is typically studied through radiotracer experiments, where a radioactive tracer is deposited as a thin layer and made to diffuse into a polycrystalline substrate. The depth profile is measured by sectioning or grinding, and counting the activity in the removed material or in the remaining substrate; it reflects a weighted average of the contributions from all boundaries. The influence of grain boundary character on diffusivity can be studied from individual boundaries using bicrystals (see examples in Refs. $[1,8,9]$ ). However, growing bicrystals of controlled misorientation is challenging, and the analysis requires an extreme sensitivity, as the amount of tracer material contained in a single boundary is very small. Furthermore, radiotracer studies most often involve mechanical sectioning or grinding, which limits the profile depth resolutions. This in turn sets a lower limit on the diffusion distance, and determines the minimum temperature at which data can be obtained. A number of studies of grain boundary diffusion of $\mathrm{Cr}$ in Ni-based, Cr-containing alloys have been reported [10-15], but data are scarce below about $600{ }^{\circ} \mathrm{C}$ (Fig. 1).

In the case of impurity diffusion, non-radioactive tracers can also be used, and depth profiles with the sensitivity required for grain boundary diffusion analysis can be obtained by ion sputtering and mass spectrometry using SIMS. This technique offers a depth resolution in the nanometer range, and thereby gives access to short diffusion distances and low temperatures. The lateral resolution of SIMS, in the tens of micrometers, also means that grain boundaries of a polycrystal can be probed individually, which potentially provides a large amount of data from a single specimen. One drawback of using stable tracers is that the substrate purity can become a limiting factor.

The present paper reports a SIMS study of Cr grain boundary diffusion in $\mathrm{Ni}$. The depth and lateral resolutions of SIMS were used to collect data at low temperatures (down to temperatures of interest for pressurized water reactor applications), and to examine the shape of individual grain boundary profiles. Measurements were also done on cold-rolled $\mathrm{Ni}$ substrates. These rapidly recrystallized during the diffusion heat treatments and provided fine-grained microstructures, from which profiles reflecting an average of several boundaries were obtained. After presenting the experimental procedures and results, the paper examines the applicability of the existing methods to process grain boundary diffusion profiles, and introduces a more general method. The grain boundary diffusivities and the remarkable features of the recorded profiles are then discussed.

This work is part of a larger effort aimed at improving the simulation of corrosion in nuclear reactors, and was intended to provide input data for a finite-difference diffusion code developed at CEA [16]. The volume diffusion of $\mathrm{Cr}$ in $\mathrm{Ni}$ and in $\mathrm{Ni}-22 \mathrm{Cr}$ was also studied [17] as part of this effort. 


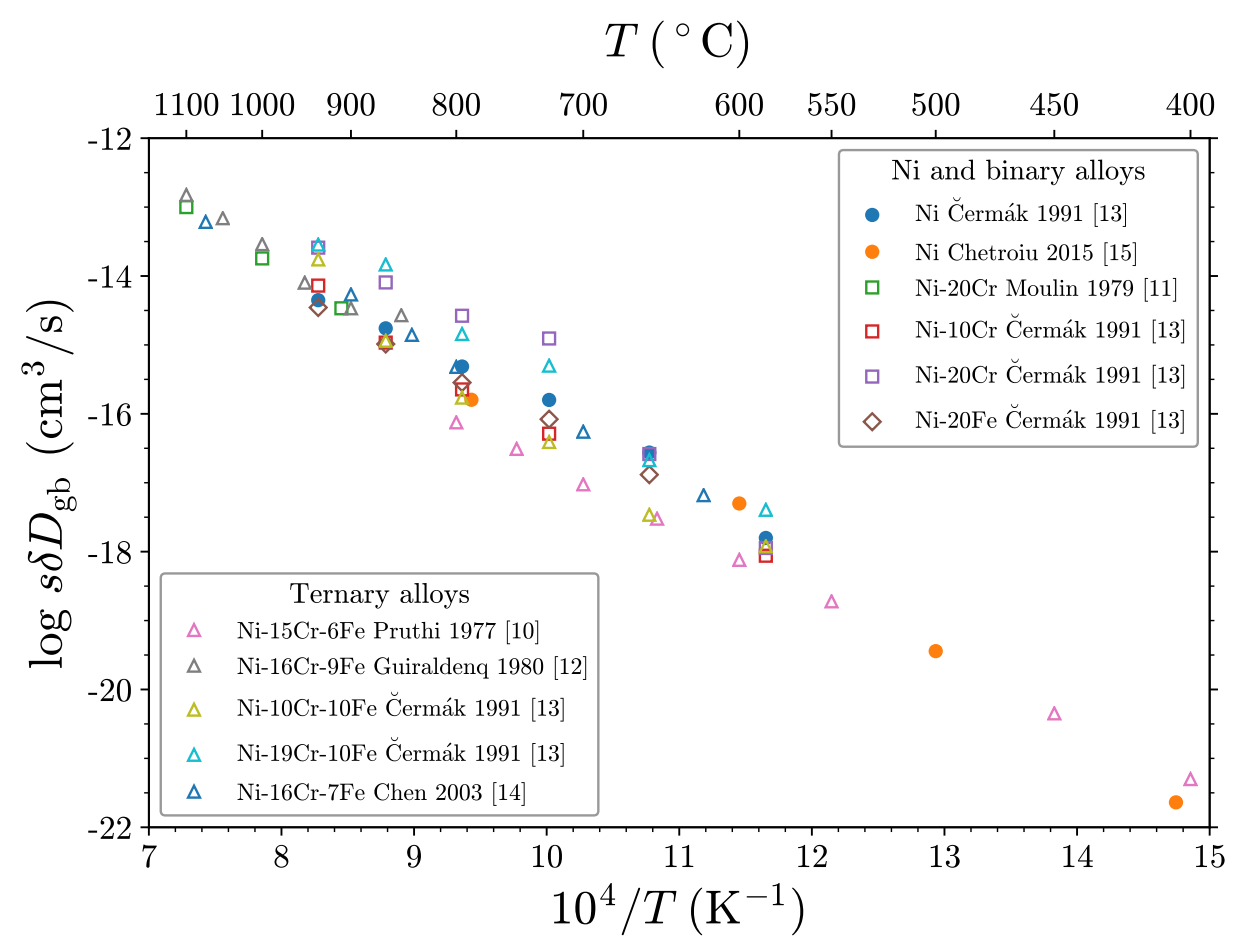

Figure 1: Grain boundary diffusivity data for $\mathrm{Cr}$ in $\mathrm{Ni}, \mathrm{Ni}-\mathrm{Cr}, \mathrm{Ni}-\mathrm{Fe}$ and $\mathrm{Ni}-\mathrm{Cr}-\mathrm{Fe}$ alloys from the literature. Measurements by radiotracer techniques, except Chetroiu [15] by SIMS. From the Čermák study [13], only the Ni-rich compositions are included here. Diffusivities are strongly affected by the presence of intergranular carbides, as shown in Refs. [11, 14], where alloys with several carbon levels were used; for these two studies, only data from the lowest carbon alloy are included here.

\section{Experimental procedures}

\subsection{Materials}

Tracer diffusion experiments were carried out using high purity Ni (5 N, Goodfellow) provided as $1 \mathrm{~mm}$ and $6 \mathrm{~mm}$ thick plates in as-rolled condition. The $1 \mathrm{~mm}$ plate was cut into $9 \times 9 \mathrm{~mm}$ coupons and subjected to a recrystallization heat treatment for $18 \mathrm{~h}$ at $1075{ }^{\circ} \mathrm{C}$ in an argon-backfilled vacuum furnace. This produced a coarse, equiaxed microstructure, with grain size approximately $1 \mathrm{~mm}$. Analysis by EBSD showed that the resulting material had no particular crystallographic texture (Fig. 2). The $6 \mathrm{~mm}$ plate was subjected to the same recrystallization heat treatment, then cold-rolled with an $86 \%$ depth reduction (strain $\epsilon=2$ ), and cut into 9 $\times 9 \mathrm{~mm}$ coupons. The resulting microstructure had a high defect density, mostly consisting of low misorientation $\left(<10^{\circ}\right)$ subgrain boundaries, as shown in Fig. 3.

The surface preparation and tracer deposition procedures were described in detail in Ref. [17]. Briefly, the specimens were ground with $\mathrm{SiC}$ paper and polished with diamond suspensions down to a $1 \mu \mathrm{m}$ finish. Thin $\mathrm{Cr}$ films were deposited by sublimation of Cr powder (99.95\% pure, Neyco) and condensation onto the polished surfaces in a vacuum chamber. A built-in quartz balance was set to obtain $5 \mathrm{~nm}$ thick films. 

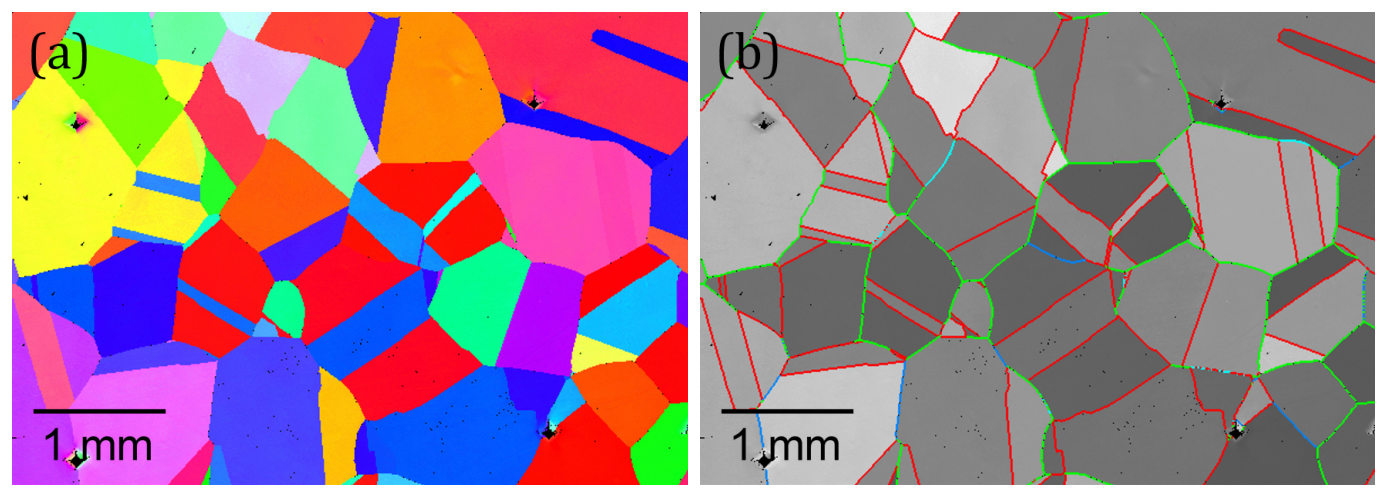

Figure 2: EBSD analysis of a $\mathrm{Ni}$ specimen annealed $18 \mathrm{~h}$ at $1075{ }^{\circ} \mathrm{C}$. (a) Inverse pole figure; (b) same figure in grayscale, with grain boundary misorientations mapped to colors: blue $<15^{\circ}$, green $15-55^{\circ}$, red $>55^{\circ}$ (red boundaries are mostly twins). (For interpretation of the references to color in this figure legend, the reader is referred to the web version of this article.)
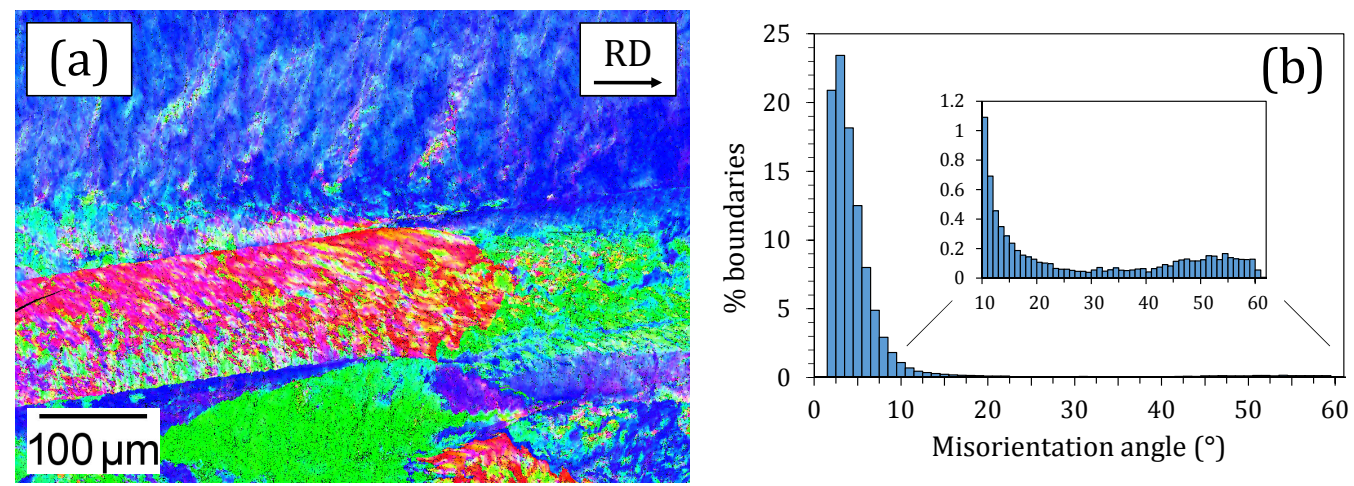

Figure 3: EBSD analysis of a cold-rolled Ni specimen ( $86 \%$ reduction). (a) Inverse pole figure; (b) misorientation angle distribution. The observations were made in the rolling direction (RD)-transverse direction plan (RD is indicated by an arrow in (a)).

\subsection{Diffusion heat treatments}

The specimens were vacuum-encapsulated in quartz capsules together with $\mathrm{Zr}$ shavings, used as oxygen getter. The diffusion heat treatments were conducted in tube furnaces. To run an experiment, a furnace was first stabilized at the desired temperature, before introducing the capsule in the hot zone. To stop an experiment, the capsule was quickly pulled from the hot zone and allowed to cool on a metal tray, reaching room temperature in less than $5 \mathrm{~min}$. Temperatures were controlled using type $\mathrm{K}$ thermocouples. The standard uncertainty was estimated to be $1.7^{\circ} \mathrm{C}$ in the temperature range of interest [17].

\subsection{SIMS analysis}

Intensity-depth profiles were measured by SIMS using a Cameca IMS $7 \mathrm{f}$ instrument. We used a duoplasmatron oxygen source with a primary current of $200 \mathrm{nA}$, collecting positive secondary ions. The raster area was $150 \times 150 \mu \mathrm{m}$, and the analysis area 
$33 \mu \mathrm{m}$ (annealed specimens) or $60 \mu \mathrm{m}$ (cold-rolled specimens) diameter. Oxygen flooding was used to reduce roughness development during sputtering.

The following isotopes were recorded: ${ }^{16} \mathrm{O},{ }^{50} \mathrm{Cr},{ }^{52} \mathrm{Cr},{ }^{54} \mathrm{Cr},{ }^{58} \mathrm{Ni}$ and ${ }^{64} \mathrm{Ni}$. Profiles were processed using the ${ }^{52} \mathrm{Cr}$ signal normalized by the ${ }^{64} \mathrm{Ni}$ signal, in order to compensate for instrument variations (normalized intensities $I\left({ }^{52} \mathrm{Cr}\right) / I\left({ }^{64} \mathrm{Ni}\right)$ are simply referred to as $I$ in the rest of the paper). A linear relation was assumed between normalized intensities and concentrations.

\subsection{Crater depth measurements}

Selected sputtering craters were analyzed by contact profilometry with a Bruker Dektak 8 instrument. Craters resulting from the analysis of a grain boundary featured a step due to the two neighboring grains being sputtered at different rates. Since the analyzed area was much smaller than the sputtered area, it was not possible to determine with reasonable accuracy what fraction of the collected signal came from one grain and the other. Depth measurements of craters made in the grains, used in the study of volume diffusion [17], gave an indication as to the variability associated with grain orientation: the sputtering rate was on average $\bar{r}_{s}=0.68 \pm 0.08$ $\mathrm{nm} / \mathrm{s}$ ( \pm one standard deviation), from 60 craters. In the study of grain boundary diffusion, all sputtering times were converted to depths using this average rate. As shown subsequently, the dispersion of grain boundary diffusivity was large, such that the error in the sputtering rate was unimportant.

\subsection{Experimental conditions}

Because they rely on approximate solutions to the diffusion equation, the methods used in the analysis of grain boundary depth profiles very much depend on the diffusion regime in which the experiments are made. Diffusion times and temperatures were chosen so as to conform to either type $\mathrm{B}$ or type $\mathrm{C}$ kinetics, according to Harrison's classification [18]. The conditions for the B regime are:

$$
s \delta \ll \sqrt{D_{\mathrm{v}} t} \ll d
$$

where $s$ is the segregation factor (ratio between the tracer concentration in a grain boundary and that in the adjacent grain), $\delta$ the grain boundary width, $D_{\mathrm{v}}$ the volume diffusion coefficient, $t$ the time and $d$ the grain size. Values of $D_{\mathrm{v}}$ were calculated from an Arrhenius law,

$$
D_{\mathrm{v}}=D_{\mathrm{v}}^{0} \exp \left(-\frac{Q_{\mathrm{v}}}{R T}\right)
$$

with $D_{\mathrm{v}}^{0}=0.2 \mathrm{~cm}^{2} / \mathrm{s}$ and $Q_{\mathrm{v}}=260 \mathrm{~kJ} / \mathrm{mol}$ taken from Ref. [17]. In Eq. (2), $R$ is the ideal gas constant and $T$ the temperature.

Volume diffusion distances were always much smaller than the grain size. For annealed substrates, this was evident given the large grain size. For cold-rolled substrates, the distance between subgrain boundaries in the as-rolled condition was not measured, but recrystallization was fast, such that diffusion essentially took place in the recrystallized state. Recrystallization kinetics were estimated by measuring the Vickers hardness (with a $0.5 \mathrm{~kg}$ mass) after annealing for various times and 
temperatures. The hardness decreased from about $2 \mathrm{GPa}$ in the as-rolled condition to about $0.7 \mathrm{GPa}$ in the recrystallized state. As an example, a value of $0.77 \mathrm{GPa}$ was measured after 20 min annealing at $460{ }^{\circ} \mathrm{C}$, which was both a shorter duration and a lower temperature than the conditions selected for experiments with coldrolled substrates. As shown subsequently, grain sizes at the end of the diffusion heat treatments were about 5 to $100 \mu \mathrm{m}$, which was far greater than $\sqrt{D_{\mathrm{v}} t}$.

With $\sqrt{D_{\mathrm{v}} t} \ll d$ true in all cases, the condition for type B kinetics may be reduced to $\alpha \ll 1$, with $\alpha$ the dimensionless parameter

$$
\alpha=\frac{s \delta}{2 \sqrt{D_{\mathrm{v}} t}}
$$

Conversely, the $\mathrm{C}$ regime corresponds to $\alpha \gg 1$. An inherent difficulty of the analysis is that the segregation factor $s$ is not known a priori; in the absence of an independent measurement, it can only be determined through the combination of type $\mathrm{B}$ and type $\mathrm{C}$ experiments [19] (the former give access to the triple product $s \delta D_{\mathrm{gb}}$, while the latter directly yield $D_{\mathrm{gb}}$ values). Analyzing the diffusion profiles therefore requires some sort of trial-and-error procedure. As noted by Gaertner et al. [19], in systems where $s>1$, experiments done with $\alpha / s>1$ can safely be assumed to fall in the $\mathrm{C}$ regime. The $\mathrm{B}$ regime threshold, on the other hand, does require $s$ to be known. In the present work, the profiles for which $\alpha / s<1$ were processed using the general solution of the diffusion equation (including contributions predominant in $\mathrm{B}$ and $\mathrm{C}$ regimes, see Section 4.2), which made the $\mathrm{B} / \mathrm{C}$ distinction less critical.

Although they did not allow $s(T)$ to be determined, the present results did suggest that in the temperature range of interest, $s$ values were not vastly different from 1 , as discussed subsequently. The experiments are therefore presented as belonging to the $\mathrm{B}$ or $\mathrm{C}$ regime based on the $\alpha / s$ values (i.e., assuming $s=1$ ). The experimental conditions are summarized in Table 1 and represented graphically in Fig. 4 (two experiments are in between the $\mathrm{B}$ and $\mathrm{C}$ regimes: these will be discussed subsequently). The experiment durations were kept above $1 \mathrm{~h}$ in order to minimize the error in the diffusion time due to diffusion occurring during heat-up and cool-down.

\section{Experimental results}

\subsection{Annealed specimens in the B regime}

The intensity-depth profiles recorded from individual grain boundaries in annealed specimens at $529-668{ }^{\circ} \mathrm{C}$ presented the general shape of type B kinetics, with a volume part steeply decaying over $100-200 \mathrm{~nm}$, and a tail reflecting accelerated diffusion along the grain boundary. Examples are shown in Fig. 5, where several profiles are included for each specimen.

The slope of the diffusion tail sometimes varied considerably between grain boundaries of a given specimen. This spread is illustrated in Fig. 5 by the two profiles recorded in the $602{ }^{\circ} \mathrm{C}$ specimen. Two other remarkable features were observed: (i) some diffusion tails contained multiple parts with markedly different slopes; (ii) some slopes were zero or even positive.

In order to examine the effect of grain boundary misorientation on diffusivity, selected specimens were analyzed by EBSD prior to $\mathrm{Cr}$ deposition and diffusion heat treatment. Profiles recorded on grain boundaries with a variety of misorientations, 
Table 1: Parameters of grain boundary diffusion experiments.

\begin{tabular}{lcccc}
\hline & $T\left({ }^{\circ} \mathrm{C}\right)$ & $t(\mathrm{~h})$ & $\sqrt{D_{\mathrm{v}} t}(\mathrm{~nm})$ & $\alpha / \mathrm{s}$ \\
\hline Annealed specimens & & & & \\
B regime & 668 & 3 & 27 & $9.1 \times 10^{-3}$ \\
& 668 & 22 & 74 & $3.4 \times 10^{-3}$ \\
& 635 & 2 & 12 & $2.0 \times 10^{-2}$ \\
& 602 & 15 & 17 & $1.4 \times 10^{-2}$ \\
& 565 & 984 & 64 & $3.9 \times 10^{-3}$ \\
& 563 & 28 & 10 & $2.4 \times 10^{-2}$ \\
C regime & 529 & 120 & 10 & $2.5 \times 10^{-2}$ \\
& 398 & 4 & $4 \times 10^{-2}$ & 6.4 \\
& 383 & 25 & $6 \times 10^{-2}$ & 4.4 \\
Rolled specimens & 360 & 87 & $5 \times 10^{-2}$ & 5.5 \\
B regime & 346 & 404 & $6 \times 10^{-2}$ & 4.5 \\
& 668 & 3 & 27 & $9.1 \times 10^{-3}$ \\
& 602 & 1 & 5 & $5.5 \times 10^{-2}$ \\
B/C regime & 573 & 4 & 5 & $5.1 \times 10^{-2}$ \\
& 546 & 23 & 6 & $3.9 \times 10^{-2}$ \\
& 515 & 96 & 6 & $4.0 \times 10^{-2}$ \\
& 515 & 1 & 1 & 0.40 \\
& 470 & 16 & 1 & 0.33 \\
\hline
\end{tabular}
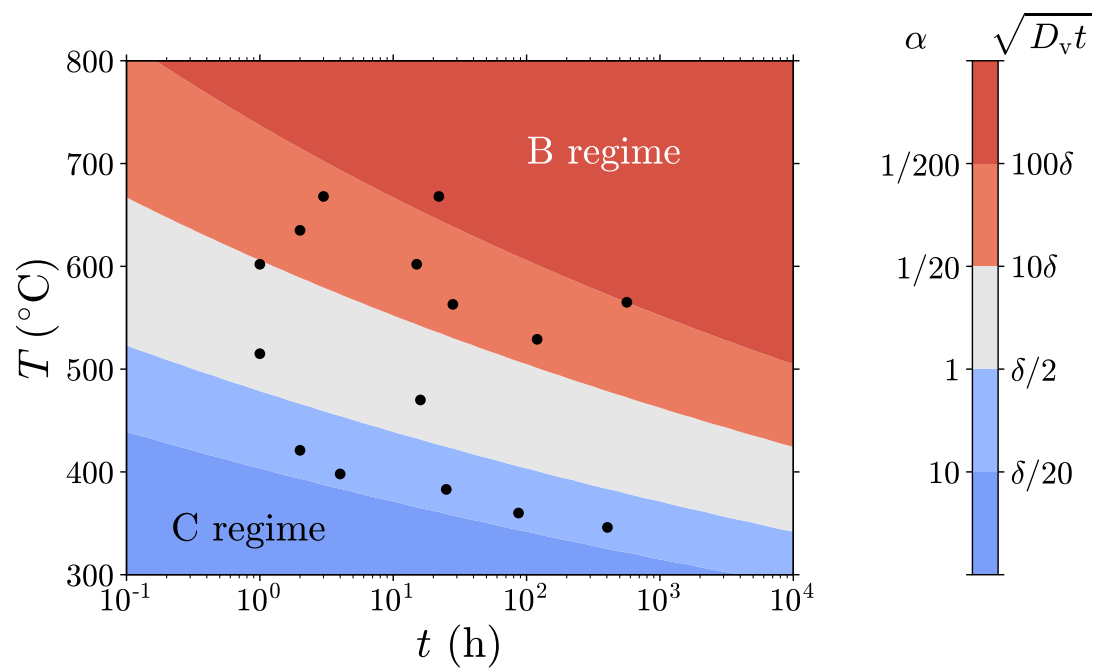

Figure 4: Map of characteristic volume diffusion distances and equivalent $\alpha$ values used in the experimental study of grain boundary diffusion (volume diffusion data from Ref. [17]). The color scale represents threshold values for the $\mathrm{B}$ and $\mathrm{C}$ regimes (see text), with $s=1$. 


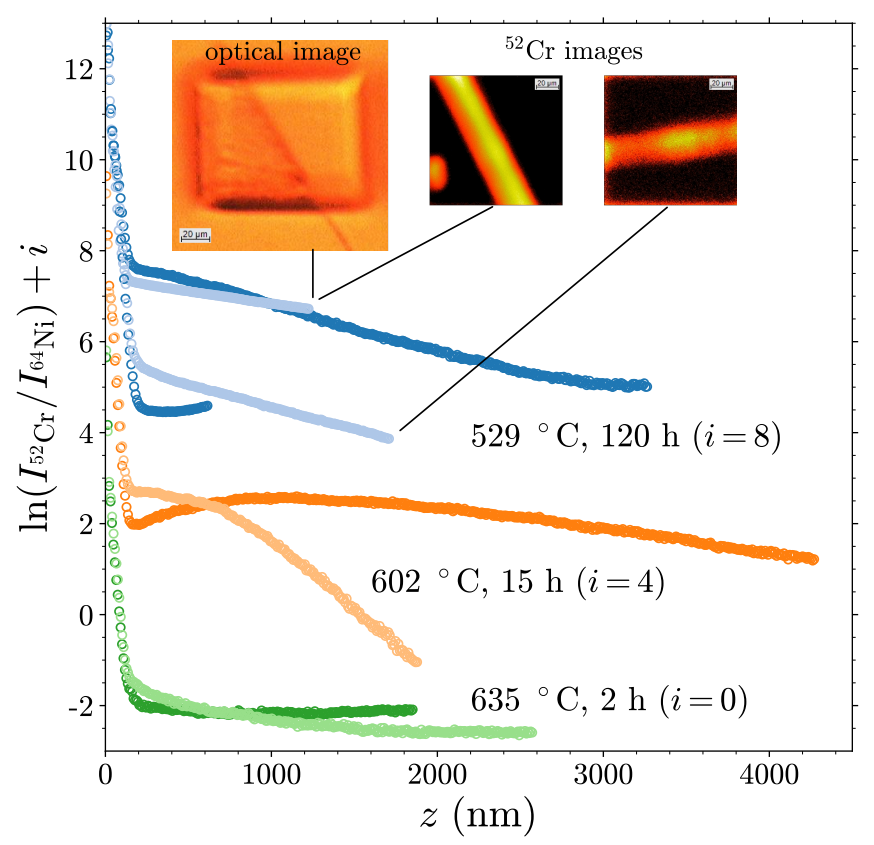

Figure 5: Examples of Cr profiles obtained from individual grain boundaries after B-regime diffusion in annealed Ni. Offsets $i$ were added to distinguish the 3 specimens. The insets show an optical image and ${ }^{52} \mathrm{Cr}$ maps recorded at the end of the indicated profiles (ion intensities mapped to colors on a linear scale). The profiles were generated from a centered, $33 \mu \mathrm{m}$ diameter area, while the maps were obtained by rastering over the $150 \times 150 \mu \mathrm{m}$ craters with a probe size of about $20 \mu \mathrm{m}$. Note that the lateral diffusion distance was much smaller than the probe size and is therefore exaggerated in the maps.

including low-angle $\left(<15^{\circ}\right)$ and high-angle $\left(>15^{\circ}\right)$, special and random, and twin boundaries $\left(60^{\circ}\right.$ misorientation around $\mathrm{a}<111>$ axis), are shown in Fig. 6 for one of these specimens, heat-treated at $563{ }^{\circ} \mathrm{C}$. The twins produced flat profiles with levels close to the background, and the ${ }^{52} \mathrm{Cr}$ maps recorded at the bottom of these craters showed no $\mathrm{Cr}$ enrichment, indicating an absence of accelerated diffusion. Of the nine profiles recorded on other types of boundaries, five had negative slopes, while two were considered flat and two had positive slopes. Given the limited number of profiles available for comparison, misorientation effects could not be evaluated.

\subsection{Rolled specimens}

Recrystallization of the cold-rolled specimens during the diffusion heat treatments was fast, and produced grains about 5 to $100 \mu \mathrm{m}$ large (see optical images and ${ }^{52} \mathrm{Cr}$ maps recorded at the bottom of sputtering craters in Fig. 7). It was then hardly possible to obtain profiles from individual grain boundaries; instead, the analysis area was increased from 33 to $60 \mu \mathrm{m}$, in an attempt to collect signal from several grain boundaries at a time.

The resulting profiles, shown in Fig. 7, had shapes similar to those obtained from annealed specimens, with the following differences: (i) the transition from 


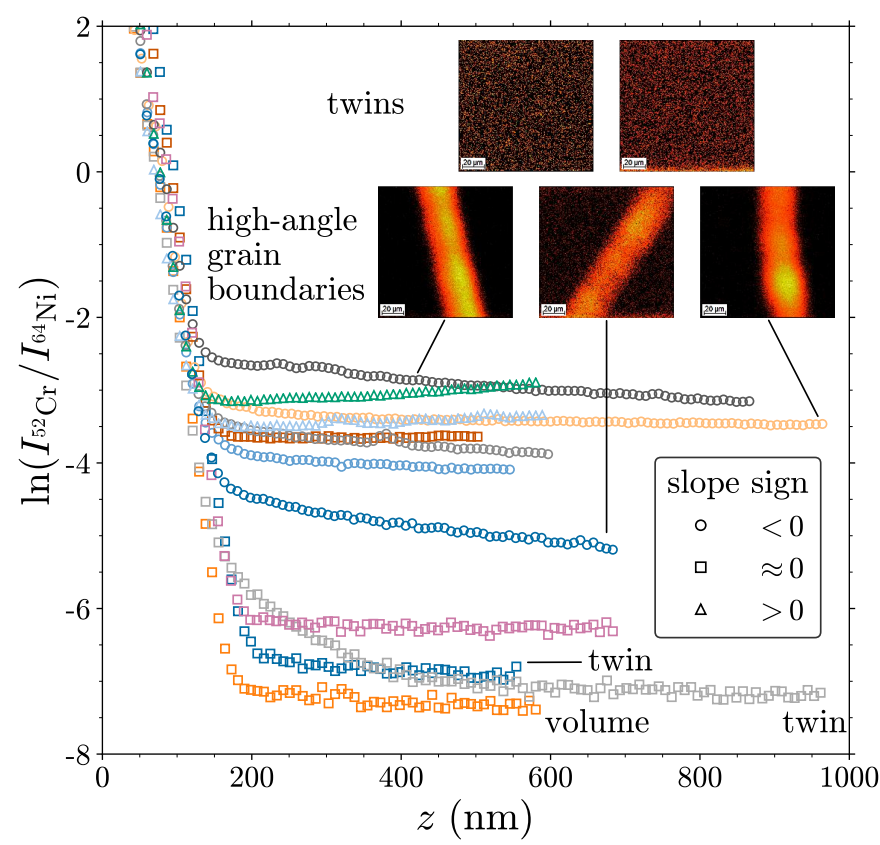

Figure 6: Chromium profiles obtained from individual grain boundaries after B-regime diffusion $\left(28 \mathrm{~h}\right.$ at $563^{\circ} \mathrm{C}$ ) in an annealed Ni specimen (probe size $\left.33 \mu \mathrm{m}\right)$. A profile recorded in a grain away from boundaries (labeled "volume") is included to indicate the background level. The insets are ${ }^{52} \mathrm{Cr}$ maps recorded at the end of the indicated profiles, showing ion intensities on a linear scale and collected with a probe size of about $20 \mu \mathrm{m}$ (the lateral diffusion distances are exaggerated due to the large probe size).

the volume part to the diffusion tail was smoother; (ii) in the diffusion tail, slope variations were less abrupt than those observed with annealed substrates; (iii) in some cases, undulations, i.e., apparently periodic slope variations, were observed.

\subsection{Annealed specimens in the $\mathrm{C}$ regime}

The profiles recorded in the annealed specimens at $346-398{ }^{\circ} \mathrm{C}$ had varying shapes - see examples in Fig. 8. In most cases, no diffusion tail was present, indicating that grain boundary diffusion did not enrich the grain boundary above the segregation level of $\mathrm{Cr}$ impurities already present in the substrate. In some cases, a tail was present but had a convex shape (decreasing slope), which is not compatible with standard solutions of the diffusion equation. Only four profiles had concave tails and could be used to determine diffusion coefficients.

\section{Data processing}

\subsection{General solution of the grain boundary diffusion problem}

The intensity-depth profiles were studied based on the analytical solution to the diffusion equation in a bicrystal with constant surface concentration by Whipple [20]. 

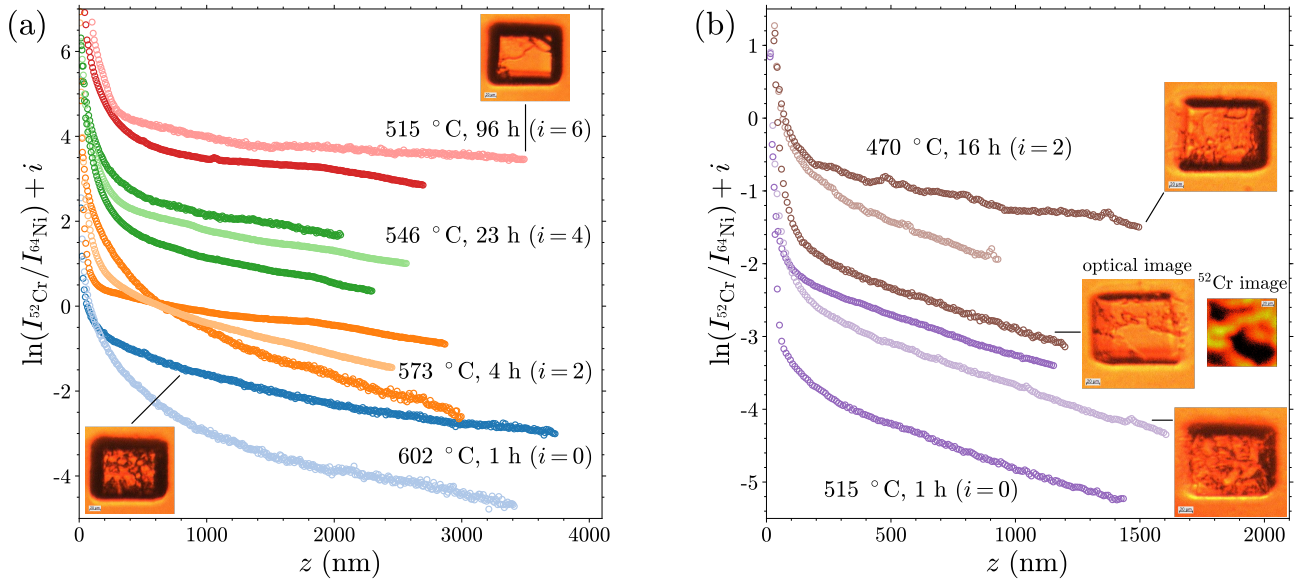

Figure 7: Chromium profiles recorded after diffusion in cold-rolled Ni (probe size $60 \mu \mathrm{m}$ ). Offsets $i$ were added to distinguish the specimens. Contrast in the optical images and the ${ }^{52} \mathrm{Cr}$ map recorded at the bottom of the sputtering craters reflects the substrate microstructure. The ion maps were generated with a probe size of about $20 \mu \mathrm{m}$, and therefore exaggerate the lateral diffusion distances. (a) $\alpha=0.01$ to 0.05 (B regime); (b) $\alpha=0.3$ and 0.4 (B/C regime).

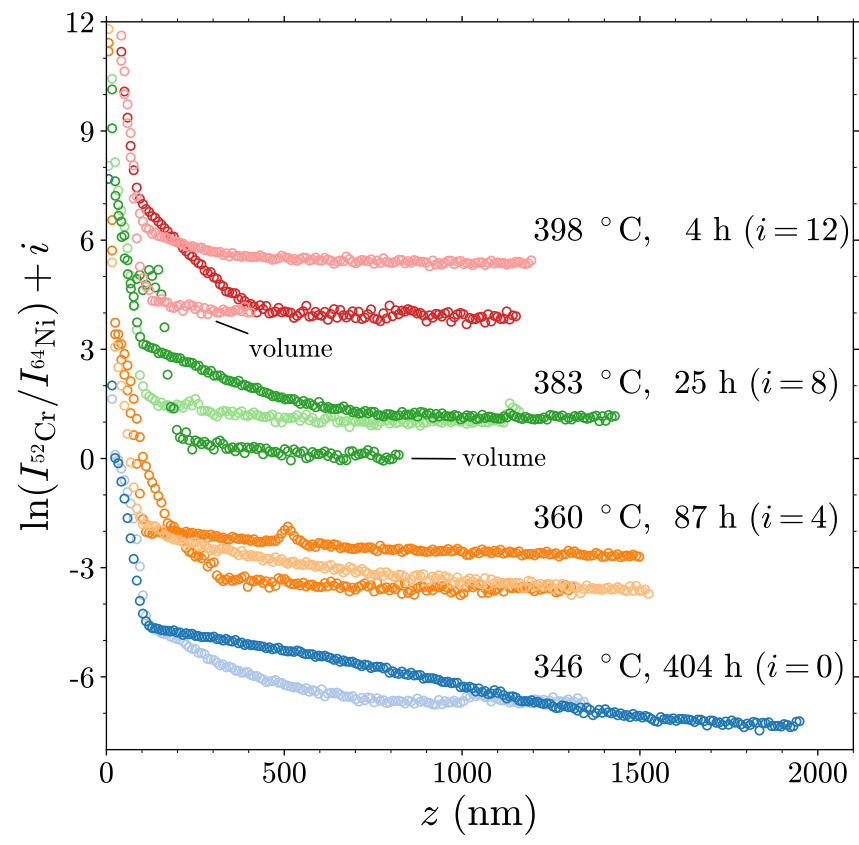

Figure 8: Chromium profiles obtained from individual grain boundaries after C-regime diffusion in annealed Ni. Offsets $i$ were added to distinguish the four specimens. 
(Due to the relatively short heat treatments/low temperatures, the deposited $\mathrm{Cr}$ was never entirely consumed, and the remaining film maintained a unit surface concentration throughout the experiments.) In a polycrystal, so long as $\sqrt{D_{\mathrm{v}} t} \ll d$, the concentration profiles generated by lateral diffusion from the grain boundaries do not overlap. In these conditions, analyzing an individual grain boundary by SIMS is equivalent to analyzing a bicrystal by the sectioning method, and the expressions obtained by integration of the two-dimensional solution can be used. (This solution corresponds to a geometry where the grain boundary is normal to the surface. The effect of the grain boundary inclination angle relative to the surface was studied by Mishin [21], and shown to be small for polycrystals with uniformly distributed angles. When analyzing a single grain boundary, the inclination can only be taken into account if the angle is measured, which was not the case here.)

A general solution, irrespective of the diffusion regime, is:

$$
C(\eta, \beta) / C_{s}=C_{1}(\eta)+C_{2}(\eta, \beta, \Delta)+C_{\mathrm{gb}}(\eta, \beta, \Delta)
$$

where $C_{s}$ is the surface concentration (here $C_{s}=1$ ) and $C_{1}, C_{2}$ and $C_{\mathrm{gb}}$ are the contributions of direct volume diffusion from the surface, of lateral diffusion from the grain boundary into the grains, and of diffusion inside the grain boundary, respectively. These are given by [8]:

$$
C_{1}(\eta)=\operatorname{erfc}(\eta / 2)
$$

$$
\begin{array}{r}
C_{2}(\eta, \beta, \Delta)=\frac{\sqrt{D_{\mathrm{v}} t}}{L} \frac{2 \eta}{\sqrt{\pi}} \int_{1}^{\Delta} \sigma^{-3 / 2} \exp \left(-\frac{\eta^{2}}{4 \sigma}\right)\left(\frac{\Delta-\sigma}{\Delta-1}\right)^{1 / 2}\left[\frac{\exp \left(-Y^{2}\right)}{\sqrt{\pi}}-Y \operatorname{erfc}(Y)\right] \mathrm{d} \sigma \\
\text { with } Y=\frac{\sigma-1}{2 \beta}\left(\frac{\Delta-1}{\Delta-\sigma}\right)^{1 / 2}, \quad(6) \\
C_{\mathrm{gb}}(\eta, \beta, \Delta)=\frac{\delta}{L} \frac{\eta}{2 \sqrt{\pi}} \int_{1}^{\Delta} \sigma^{-3 / 2} \exp \left(-\frac{\eta^{2}}{4 \sigma}\right) \operatorname{erfc}\left[\frac{\sigma-1}{2 \beta}\left(\frac{\Delta-1}{\Delta-\sigma}\right)^{1 / 2}\right] \mathrm{d} \sigma
\end{array}
$$

In Eqs. (5-7), $\eta$ is the reduced variable for depth,

$$
\eta=\frac{z}{\sqrt{D_{\mathrm{v}} t}}
$$

(with $z$ the depth), $\beta$ is a dimensionless parameter reflecting the sharpness of the concentration contours,

$$
\eta=\frac{D_{\mathrm{gb}}}{D_{\mathrm{v}}} \frac{s \delta}{2 \sqrt{D_{\mathrm{v}} t}},
$$

$D_{\mathrm{gb}}$ is the grain boundary diffusion coefficient, $\Delta=D_{\mathrm{gb}} / D_{\mathrm{v}}$ and $L$ is the analysis length normal to the grain boundary. 


\subsection{Method for B-type profiles}

In the $\mathrm{B}$ regime $(\alpha \ll 1)$, the contribution of tracer atoms inside the grain boundary (the $C_{\mathrm{gb}}$ term) can be neglected, and $C_{2}$ can be approximated by [22]:

$$
\begin{array}{r}
C_{2}(\eta, \beta, \Delta)=\frac{\sqrt{D_{\mathrm{v}} t}}{L} \frac{2 \eta}{\sqrt{\pi}} \int_{1}^{\infty} \sigma^{-3 / 2} \exp \left(-\frac{\eta^{2}}{4 \sigma}\right)\left[\frac{\exp \left(-Y^{2}\right)}{\sqrt{\pi}}-Y \operatorname{erfc}(Y)\right] \mathrm{d} \sigma \\
\text { with } Y=\frac{\sigma-1}{2 \beta}
\end{array}
$$

Even approximated, the analytical form of $C_{2}$ does not lend itself to linearization, in view of fitting an experimental profile. The problem is usually addressed as follows.

The following relation can be derived from the definition of $\eta$ and $\beta$ [22]:

$$
s \delta D_{\mathrm{gb}}=\left(-\frac{\partial \ln C}{\partial z^{n}}\right)^{-2 / n}\left(\frac{4 D_{\mathrm{v}}}{t}\right)^{1 / 2}\left(-\frac{\partial \ln C}{\partial\left(\eta \beta^{-1 / 2}\right)^{n}}\right)^{2 / n}
$$

where $n$ is any nonzero number. Eq. (11) holds regardless of the diffusion conditions, i.e., of the shape taken by $C$ : it only expresses the relationship between gradients of $\ln C$ plotted against $z^{n}$ and against the dimensionless number $\left(\eta \beta^{-1 / 2}\right)^{n}$. However, for $C_{2}$ given by Eq. (10), within some conditions, the gradient $A_{n}=-\frac{\partial \ln C}{\partial\left(\eta \beta^{-1 / 2}\right)^{n}}$ is practically independent of $\beta$ and varies slowly with $\eta \beta^{-1 / 2}$, such that it can be considered independent of the experimental conditions. This property is usually used to determine the triple product $s \delta D_{\mathrm{gb}}$. Fischer [23] used an approximate solution to the diffusion equation to derive $A_{1}=\pi / 4$. Following on the work of Levine and MacCollum [24], Le Claire [22] showed that for $\beta \gg 1, A_{6 / 5}=0.78$ within a few percents, i.e., to a better approximation than Fischer's $A_{1}$. Profiles are then typically studied from a $\left(z^{6 / 5}, \ln I\right)$ plot, and $s \delta D_{\mathrm{gb}}$ is calculated using the slope of the linear tail in Eq. (11), with $n=6 / 5$ and $A_{6 / 5}=0.78$ (in practice, the measured quantity is an intensity proportional to the tracer concentration, such that the experimental slope $\frac{\partial \ln I}{\partial z^{6 / 5}}$ is used in place of $\frac{\partial \ln C}{\partial z^{6 / 5}}$ in Eq. (11)).

The properties of $A_{n}$ gradients computed from $C$ given by Eqs. (4-7) in the conditions of the present study are studied in the Appendix. Examination shows that Le Claire's method is not well suited to the present experiments, for several reasons. First, $A_{6 / 5}$ is constant to a good approximation only for $\eta \beta^{-1 / 2}$ greater than about 2. This is usually the case in experiments done with a sectioning or grinding method at high temperature, where $\eta \beta^{-1 / 2}$ values typically range from 4 to 8 . Here, the depths that could be analyzed by SIMS were limited to a few $\mu \mathrm{m}$, and the diffusion times had to be long enough to remain in the $\mathrm{B}$ regime - this produced small $\eta$ values. Furthermore, because of the low temperature, the $\beta$ values were relatively large. These constraints resulted in $\eta \beta^{-1 / 2}$ values mostly between 0.1 and 1 (Table 1 ).

Second, $\alpha$ values were relatively large for the B regime, such that Eq. (10) was not a good approximation of $C_{2}$. At a given temperature, $\alpha$ can be reduced by increasing the diffusion time. Here, the diffusion times were kept relatively short in an attempt to keep the slopes of the diffusion tails large enough to be measured this was an issue because of the relatively high $D_{\mathrm{gb}} / D_{\mathrm{v}}$ ratio at the temperatures 
of interest. The variations of $A_{6 / 5}$ and the error that would be made by using a constant $A_{6 / 5}=0.78$ are detailed in the Appendix.

Finally, another factor is that when a diffusion tail contains several linear parts (which was sometimes the case here, see Section 3.1), the boundary conditions pertaining to the second (and subsequent) part(s) of the tail are not those underlying Eq. (4). If, for example, grain boundary diffusion is first very fast and then much slower from depth $z_{0}$, the diffusion equation for $z>z_{0}$ would be most conveniently solved by considering a constant concentration at $z_{0}$. With Le Claire's method, one would then need to work from a $\left(\left(z-z_{0}\right)^{6 / 5}, \ln I\right)$ plot, since the $z^{6 / 5}$ transform would not conserve the slope. This can be avoided by using a $(z, \ln I)$ plot $(n=1)$ [25], but an appropriate $A_{1}$ gradient has to be determined for every profile.

In order to overcome these issues, the profiles were analyzed using the general solution, Eqs. (4-7). For each profile, $s \delta D_{\mathrm{gb}}$ was obtained as the root of the function

$$
f: s \delta D_{\mathrm{gb}} \rightarrow\left(\frac{\partial \ln C}{\partial z}\right)^{\mathrm{calc}}-\left(\frac{\partial \ln I}{\partial z}\right)^{\exp },
$$

where $\left(\frac{\partial \ln I}{\partial z}\right)^{\exp }$ is the slope obtained from a linear least-squares fit of the tail part on an experimental $(z, \ln I)$ plot, and $\left(\frac{\partial \ln C}{\partial z}\right)^{\text {calc }}$ is the gradient calculated from Eqs. (4-7) and averaged over the $z$ range used for fitting. This optimization problem is nonlinear, but if $D_{\mathrm{v}}$ is known, in a given $z$ range, $\frac{\partial \ln C}{\partial z}$ is a function of $s \delta D_{\mathrm{gb}}$ only, and the root can be computed numerically. This was done using functions of the Scipy [26] Python package, with $s \delta D_{\text {gb }}$ extrapolated from Čermák's data [13] as an initial guess. Values of $D_{\mathrm{v}}$ were calculated from Ref. [17]. The script is given in Supplementary material S1, and an example profile in Supplementary Material S2.

Note that since an exact $\frac{\partial \ln C}{\partial z}$ gradient is computed, the choice of a $(z, \ln I)$ or a $\left(z^{6 / 5}, \ln I\right)$ plot $(n=1$ or $n=6 / 5)$ has no bearing on the final result provided the slope correctly represents the data, i.e., provided that the experimental gradient is relatively constant. In practice, the diffusion tails were no less linear with $(z, \ln I)$ coordinates - the correlation factor of the linear fit $R^{2}$ was in fact slightly greater with $n=1$, compared to $n=6 / 5$. The calculations in the Appendix show that $A_{1}$ is indeed more constant than $A_{6 / 5}$ for small $\eta \beta^{-1 / 2}$ values. In addition, $(z, \ln I)$ coordinates have the advantage that they give the same weight to all data points, and are therefore better suited to tails with multiple parts.

It is further noted that in using the general solution, Eqs. (4-7), the present method makes no assumption as to the diffusion regime. In this respect, it is equivalent to the fitting procedure by Shaw and Shaw [27]. However, in Ref. [27], $s \delta D_{\mathrm{gb}}$ is directly computed from a non-linear fit of the experimental data, whereas here, a linear fit is first performed before computing $s \delta D_{\mathrm{gb}}$ from the adjusted slope this of course is only applicable in conditions where the diffusion tail is a straight line. The rationale behind this apparent limitation is that in addition to slope variations expected from Eqs. (4-7), curvature in a diffusion profile can arise due to other causes, such as diffusivity variations. This is particularly true with profiles measured on individual grain boundaries, as shown in Section 3. In this context, a non-linear fit could produce errors, while restraining the analysis to the linear part of the diffusion tails (and treating C-regime profiles separately) represents a more conservative approach. 
The applicability range of Le Claire's method and the question of linearity in diffusion tails have been studied by other authors, e.g., Gryaznov et al. [28], who proposed a processing method designed for profiles with short diffusion distances (large $\alpha$ ). In Ref. [28], $s \delta D_{\text {gb }}$ is calculated using a relationship derived from Eq. (11), which involves the maximum value of the experimental $\frac{\partial \ln C}{\partial z^{6 / 5}}$ gradient, and the minimum value of the $A_{6 / 5}$ gradient. The latter is provided as a function of $\alpha, A_{6 / 5}=$ $0.71+0.77 \alpha$. This has the advantage of improving the accuracy of the calculated $s \delta D_{\mathrm{gb}}$, compared to Le Claire's $A_{6 / 5}=0.78$, while avoiding the computation of the exact solution. However, the relationship giving $A_{6 / 5}$ was obtained from the solution in the form $C=C_{1}+C_{2}$, where $C_{\mathrm{gb}}$ was omitted. Although the method was intended for the $\mathrm{B}$ regime, it was specifically targeted at profiles with relatively large $\alpha$ values (above 0.02), conditions in which $C_{\mathrm{gb}}$ starts to have a significant influence, as shown in the Appendix. While also intended for the $\mathrm{B}$ regime, the present method does take into account the contribution of $C_{\mathrm{gb}}$, and thus also covers the start of the $\mathrm{B} / \mathrm{C}$ transition, where diffusion tails still display near linearity.

\subsection{Application to B-type profiles}

Negative slopes could be determined with reasonable confidence down to an absolute value of about $10^{-4} \mathrm{~nm}^{-1}$. Below this, the correlation coefficient $R^{2}$ dropped, and the slope varied significantly when changing the fitting interval, indicating an absence of correlation: these profiles were considered flat (slope $\approx 0$ ) and dismissed. The profiles with positive slopes were also not used to determine diffusivities. The $s \delta D_{\mathrm{gb}}$ values obtained according to the above method from the negative slopes of the B-type profiles recorded in annealed specimens (Figs. 5 and 6) are given in Table 2. Values obtained from different grain boundaries of a given specimen were found to differ by factors of up to 75 .

As noted in Section 3.2, the profiles recorded in rolled specimens displayed a smooth transition from the volume part to the diffusion tail and an apparent periodicity in the slope of the tail. As a consequence, the depth range to be used for fitting could not be identified unambiguously from a visual examination of the profiles. A local fitting procedure was then used, where instead of fitting the data in a previously identified range, the fit is done in a small translating interval. This amounts to computing a moving average of the slope $\frac{\partial \ln I}{\partial z}$, in a manner analogous to the analysis of oxidation mass-gain curves in Ref. [29]. Here, the evolution of the local slope gives indications as to whether and where the profile is linear. The optimal interval width and translation step depend on the amplitude of the slope variations and on the signal-to-noise ratio. Tests led us to choose a $200 \mathrm{~nm}$ width and a $30 \mathrm{~nm}$ step.

This local analysis further emphasized the variety in the shape of the diffusion tails. Four examples are shown in Fig. 9, where in each case the intensity and local slope profiles are superimposed. In ranges where the local slope was found to be stable, an average slope was determined from a least-squares fit: this is shown by the orange lines. Fig. 9(a) and (b) illustrate two types of signal variations. In both cases, the slope gradually decreases (in absolute value) before stabilizing. However, in (a) the signal appears to be subject to random noise, while in (b) the frequency of background variations appears to be lower and less widely distributed - this could indicate actual diffusivity variations associated with the finely recrystallized 
Table 2: Grain boundary diffusion parameters obtained from annealed specimens in the B regime. The slopes were measured from $(z, \ln I)$ plots. The $\eta$ and $\eta \beta^{-1 / 2}$ values are given at midpoint of the $z$ range used for fitting.

\begin{tabular}{ccccccc}
\hline \multirow{2}{*}{$T\left({ }^{\circ} \mathrm{C}\right)$} & $\alpha$ & $\beta$ & \multicolumn{2}{c}{ Midpoint } & Slope $\left(\mathrm{nm}^{-1}\right)$ & $s \delta D_{\mathrm{gb}}\left(\mathrm{cm}^{3} / \mathrm{s}\right)$ \\
\cline { 3 - 5 } & & & $\eta$ & $\eta \beta^{-1 / 2}$ & & \\
\hline 668 & $9.1 \times 10^{-3}$ & $1.4 \times 10^{3}$ & 26 & 0.68 & $-1.0 \times 10^{-3}$ & $5.4 \times 10^{-18}$ \\
668 & $3.4 \times 10^{-3}$ & $1.3 \times 10^{2}$ & 18 & 1.6 & $-1.3 \times 10^{-3}$ & $1.3 \times 10^{-18}$ \\
635 & $2.0 \times 10^{-2}$ & $3.1 \times 10^{4}$ & 81 & 0.46 & $-4.7 \times 10^{-4}$ & $1.6 \times 10^{-17}$ \\
602 & $1.4 \times 10^{-2}$ & $1.4 \times 10^{4}$ & 190 & 1.6 & $-5.4 \times 10^{-4}$ & $2.7 \times 10^{-18}$ \\
& & $5.1 \times 10^{2}$ & 83 & 3.7 & $-3.1 \times 10^{-3}$ & $1.0 \times 10^{-19}$ \\
565 & $3.9 \times 10^{-3}$ & $5.5 \times 10^{3}$ & 26 & 0.36 & $-2.1 \times 10^{-4}$ & $8.3 \times 10^{-19}$ \\
& & $1.5 \times 10^{3}$ & 43 & 1.1 & $-4.4 \times 10^{-4}$ & $2.2 \times 10^{-19}$ \\
563 & $2.4 \times 10^{-2}$ & $7.1 \times 10^{3}$ & 48 & 0.57 & $-1.2 \times 10^{-3}$ & $1.6 \times 10^{-19}$ \\
& & $1.5 \times 10^{4}$ & 53 & 0.43 & $-8.0 \times 10^{-4}$ & $3.4 \times 10^{-19}$ \\
& & $1.8 \times 10^{4}$ & 41 & 0.30 & $-7.2 \times 10^{-4}$ & $4.1 \times 10^{-19}$ \\
& & $5.5 \times 10^{5}$ & 77 & 0.10 & $-1.3 \times 10^{-4}$ & $1.2 \times 10^{-17}$ \\
& & $3.4 \times 10^{4}$ & 36 & 0.20 & $-5.2 \times 10^{-4}$ & $7.7 \times 10^{-19}$ \\
529 & $2.5 \times 10^{-2}$ & $9.1 \times 10^{3}$ & 110 & 1.2 & $-1.1 \times 10^{-3}$ & $4.0 \times 10^{-20}$ \\
& & $1.6 \times 10^{4}$ & 210 & 1.7 & $-9.1 \times 10^{-4}$ & $6.9 \times 10^{-20}$ \\
& & $3.8 \times 10^{4}$ & 81 & 0.42 & $-5.4 \times 10^{-4}$ & $1.7 \times 10^{-19}$ \\
& & $1.2 \times 10^{4}$ & 110 & 1.0 & $-9.8 \times 10^{-4}$ & $5.4 \times 10^{-20}$ \\
\hline & & & & & &
\end{tabular}

microstructure. In each of (c) and (d), two distinct intervals are separated by an abrupt transition. In both cases, after the transition, the slope is found to be stable. However, before the transition, the slope had stabilized in case (c), while it kept decreasing in $(\mathrm{d})$.

Stable slope values determined this way were used to determine triple products according to the above method. These $s \delta D_{\mathrm{gb}}$ values are given in Table 3 .

\subsection{Processing C-type profiles}

Type $\mathrm{C}$ kinetics describe a regime where no diffusion takes place in the volume, either directly from the surface or laterally from the grain boundaries, i.e., in the limit of $\sqrt{D_{\mathrm{v}} t} \rightarrow 0$. The diffusion problem can then be studied with solutions equivalent to those applying in homogeneous media, considering only the tracer present in the grain boundary [8]. The error made by assuming type $\mathrm{C}$ kinetics in practical cases, where $\sqrt{D_{\mathrm{v}} t}$ is necessarily non-zero, depends on the accuracy that can be expected given experimental uncertainties. Kaur et al. [8] recommend $\alpha>10$, and add that the error on $D_{\mathrm{gb}}$ is reduced to a few percents for $\alpha>3$. Paul et al. [30] recommend $\alpha>1$.

Here, $\alpha / s$ values were in the range 4 to 6 (Table 1 ). In these conditions, $C_{1}$ and $C_{2}$ can safely be neglected, and $C$ is well approximated by:

$$
\frac{C(z, t)}{C_{s}}=\frac{\delta}{L} \operatorname{erfc}\left(\frac{z}{\sqrt{D_{\mathrm{gb}} t}}\right)
$$



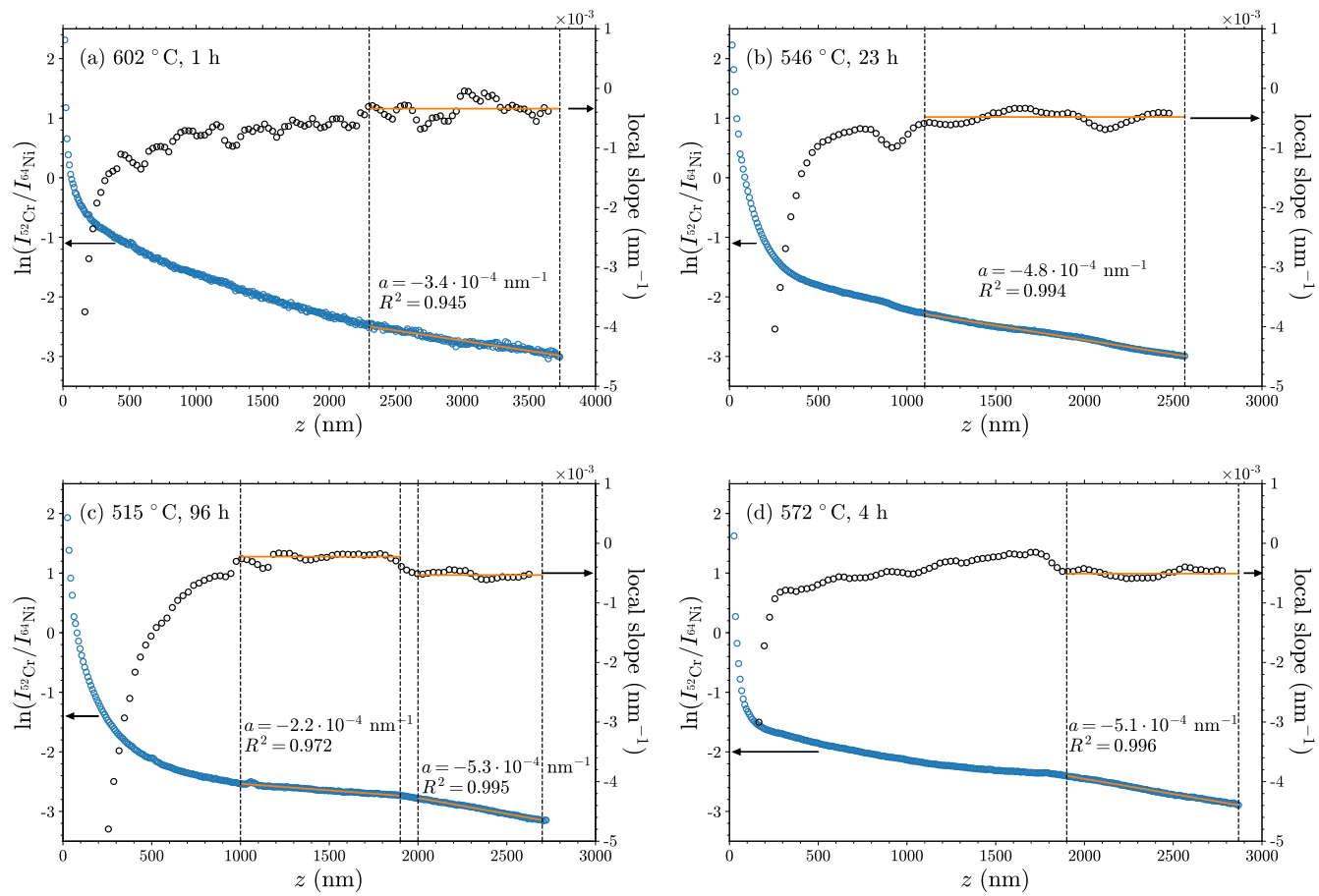

Figure 9: Analysis of $\mathrm{Cr}$ profiles recorded in cold-rolled Ni using a local fitting procedure (see text). The profile selection illustrates the variety of shapes encountered in the study of cold-rolled specimens.

Equation (12) is written in terms of concentration, but the experimental profiles were available in terms of normalized intensities, which were assumed to be proportional to concentrations. Since the inverse complementary error function does not transform products into sums, linearizing Eq. (12) requires knowledge of the proportionality constant. The latter can also be found by iteration, as was done here. A function $f: z \rightarrow a z+b$ was fitted to the data in the form $\left(z, \operatorname{erfc}^{-1}(k \cdot I)\right)$. The appropriate $k$ value was obtained by minimizing $|b|$, which provided a linear plot. The slope a was determined from a least-squares fit, and $D_{\mathrm{gb}}$ obtained as $D_{\mathrm{gb}}=\left(4 a^{2} t\right)^{-1}$. The values obtained this way from the C-type profiles with concave tails (see Section 3.3) are given in Table 4.

\section{Discussion}

\subsection{Comparison of $\mathrm{B}$ and $\mathrm{C}$ regime results}

Values of $s \delta D_{\mathrm{gb}}$ and $D_{\mathrm{gb}}$ obtained from B and C regime measurements, respectively, can be combined to determine $s$ or $\delta$, if the other is known. Divinski et al. [31] used this method with self-diffusion experiments $(s=1)$ and obtained $\delta=0.54 \mathrm{~nm}$ in high purity $\mathrm{Ni}$, in agreement with the commonly accepted value of $\delta=0.5 \mathrm{~nm}$ in fcc metals [8].

The $s \delta D_{\mathrm{gb}}$ values measured here in the $\mathrm{B}$ regime are gathered in Fig. 10. Products $\delta D_{\mathrm{gb}}$ derived from $D_{\mathrm{gb}}$ measured in the C regime assuming $\delta=0.5 \mathrm{~nm}$ are also plotted. The vertical offset between the two data series represents the amplitude 
Table 3: Grain boundary diffusion parameters obtained from the cold-rolled specimens. The slopes were measured from $(z, \ln I)$ plots. The $\eta$ and $\eta \beta^{-1 / 2}$ values are given at midpoint of the $z$ range used for fitting.

\begin{tabular}{|c|c|c|c|c|c|c|}
\hline \multirow[t]{2}{*}{$T\left({ }^{\circ} \mathrm{C}\right)$} & \multirow[t]{2}{*}{$\alpha$} & \multirow[t]{2}{*}{$\beta$} & \multicolumn{2}{|c|}{ Midpoint } & \multirow[t]{2}{*}{ Slope $\left(\mathrm{nm}^{-1}\right)$} & \multirow[t]{2}{*}{$s \delta D_{\mathrm{gb}}\left(\mathrm{cm}^{3} / \mathrm{s}\right)$} \\
\hline & & & $\eta$ & $\eta \beta^{-1 / 2}$ & & \\
\hline 668 & $9.1 \times 10^{-3}$ & $6.1 \times 10^{2}$ & 73 & 3.0 & $-1.8 \times 10^{-3}$ & $2.3 \times 10^{-18}$ \\
\hline \multirow[t]{2}{*}{602} & $5.5 \times 10^{-2}$ & $4.9 \times 10^{5}$ & 670 & 0.95 & $-3.4 \times 10^{-4}$ & $2.5 \times 10^{-17}$ \\
\hline & & $1.8 \times 10^{5}$ & 560 & 1.3 & $-5.7 \times 10^{-4}$ & $9.2 \times 10^{-18}$ \\
\hline \multirow[t]{3}{*}{573} & $5.1 \times 10^{-2}$ & $6.8 \times 10^{4}$ & 450 & 1.7 & $-8.9 \times 10^{-4}$ & $1.1 \times 10^{-18}$ \\
\hline & & $1.9 \times 10^{5}$ & 490 & 1.1 & $-5.1 \times 10^{-4}$ & $3.1 \times 10^{-18}$ \\
\hline & & $9.6 \times 10^{4}$ & 370 & 1.2 & $-7.3 \times 10^{-4}$ & $1.5 \times 10^{-18}$ \\
\hline \multirow[t]{4}{*}{546} & $3.9 \times 10^{-2}$ & $4.5 \times 10^{4}$ & 130 & 0.62 & $-7.6 \times 10^{-4}$ & $2.9 \times 10^{-19}$ \\
\hline & & $9.1 \times 10^{4}$ & 270 & 0.90 & $-5.5 \times 10^{-4}$ & $5.9 \times 10^{-19}$ \\
\hline & & $1.2 \times 10^{5}$ & 280 & 0.83 & $-4.8 \times 10^{-4}$ & $7.7 \times 10^{-19}$ \\
\hline & & $7.7 \times 10^{4}$ & 260 & 0.92 & $-6.0 \times 10^{-4}$ & $5.0 \times 10^{-19}$ \\
\hline \multirow[t]{4}{*}{515} & $4.0 \times 10^{-2}$ & $6.8 \times 10^{4}$ & 140 & 0.56 & $-6.5 \times 10^{-4}$ & $9.4 \times 10^{-20}$ \\
\hline & & $1.0 \times 10^{6}$ & 410 & 0.41 & $-1.7 \times 10^{-4}$ & $1.4 \times 10^{-18}$ \\
\hline & & $5.4 \times 10^{5}$ & 230 & 0.32 & $-2.2 \times 10^{-4}$ & $7.6 \times 10^{-19}$ \\
\hline & & $1.1 \times 10^{5}$ & 380 & 1.2 & $-5.3 \times 10^{-4}$ & $1.5 \times 10^{-19}$ \\
\hline \multirow[t]{3}{*}{515} & 0.40 & $3.5 \times 10^{6}$ & 1300 & 0.71 & $-1.0 \times 10^{-3}$ & $4.9 \times 10^{-19}$ \\
\hline & & $2.7 \times 10^{6}$ & 1400 & 0.87 & $-1.2 \times 10^{-3}$ & $3.8 \times 10^{-19}$ \\
\hline & & $3.0 \times 10^{6}$ & 1700 & 0.96 & $-1.1 \times 10^{-3}$ & $4.2 \times 10^{-19}$ \\
\hline \multirow[t]{3}{*}{470} & 0.33 & $1.4 \times 10^{6}$ & 820 & 0.69 & $-1.3 \times 10^{-3}$ & $2.1 \times 10^{-20}$ \\
\hline & & $2.5 \times 10^{6}$ & 1300 & 0.80 & $-9.9 \times 10^{-4}$ & $3.7 \times 10^{-20}$ \\
\hline & & $7.0 \times 10^{6}$ & 1200 & 0.46 & $-5.6 \times 10^{-4}$ & $1.0 \times 10^{-19}$ \\
\hline
\end{tabular}

Table 4: Grain boundary diffusion parameters obtained from annealed specimens in the C regime. The slopes were measured from $\left(z, \operatorname{erfc}^{-1}(k \cdot I)\right)$ plots. The $\eta$ and $\eta \beta^{-1 / 2}$ values are given at midpoint of the $z$ range used for fitting.

\begin{tabular}{|c|c|c|c|c|c|c|}
\hline \multirow[t]{2}{*}{$T\left({ }^{\circ} \mathrm{C}\right)$} & \multirow[t]{2}{*}{$\alpha$} & \multirow[t]{2}{*}{$\beta$} & \multicolumn{2}{|c|}{ Midpoint } & \multirow[t]{2}{*}{ Slope $\left(\mathrm{nm}^{-1}\right)$} & \multirow[t]{2}{*}{$D_{\mathrm{gb}}\left(\mathrm{cm}^{2} / \mathrm{s}\right)$} \\
\hline & & & $\eta$ & $\eta \beta^{-1 / 2}$ & & \\
\hline \multirow[t]{2}{*}{398} & 6.4 & $4.7 \times 10^{7}$ & $4.0 \times 10^{3}$ & 0.58 & $4.7 \times 10^{-3}$ & $7.9 \times 10^{-15}$ \\
\hline & & $2.5 \times 10^{8}$ & $5.0 \times 10^{3}$ & 0.32 & $2.0 \times 10^{-3}$ & $4.2 \times 10^{-14}$ \\
\hline 383 & 4.4 & $6.8 \times 10^{7}$ & $3.5 \times 10^{3}$ & 0.43 & $2.2 \times 10^{-3}$ & $5.6 \times 10^{-15}$ \\
\hline 346 & 4.5 & $4.2 \times 10^{8}$ & $1.3 \times 10^{4}$ & 0.61 & $9.2 \times 10^{-4}$ & $2.0 \times 10^{-15}$ \\
\hline
\end{tabular}




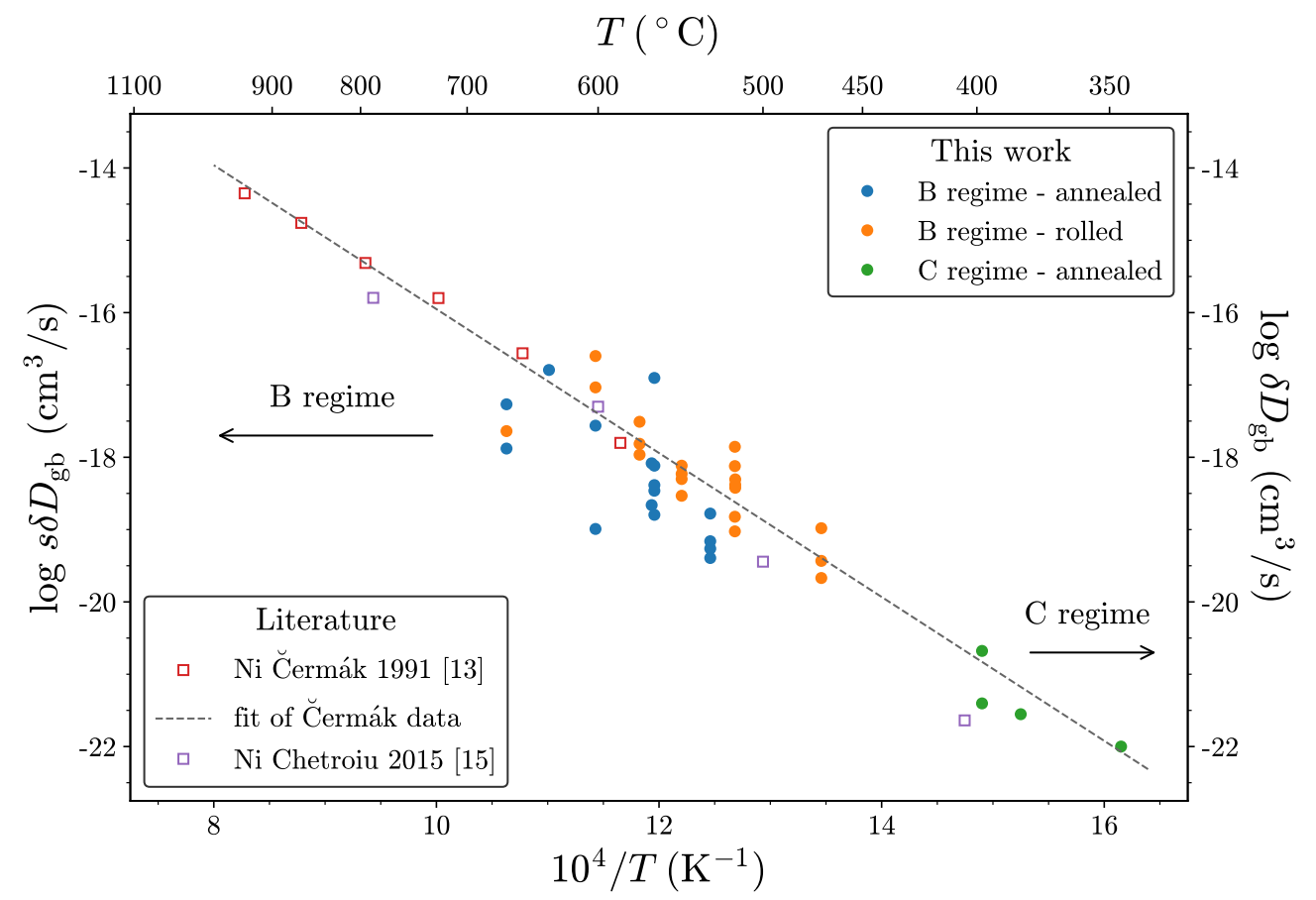

Figure 10: Triple products $s \delta D_{\mathrm{gb}}$ obtained for $\mathrm{Cr}$ diffusion in annealed and cold-rolled Ni substrates in the $\mathrm{B}$ regime. Values of $\delta D_{\mathrm{gb}}$ obtained from $D_{\mathrm{gb}}$ in the $\mathrm{C}$ regime, assuming $\delta=0.5 \mathrm{~nm}$, were added for comparison.

of the segregation factor. Specifically, if the temperature dependence of each data series can be described by an Arrhenius law, the temperature-dependent $s$ is to be calculated from:

$$
s(T)=\frac{1}{\delta} \frac{\left(s \delta D_{\mathrm{gb}}\right)^{B}}{\left(D_{\mathrm{gb}}\right)^{C}}=\frac{1}{\delta} \frac{P_{0}^{B}}{D_{0}^{C}} \exp \left(-\frac{Q^{B}-Q^{C}}{R T}\right)
$$

where $P_{0}^{B}$ and $D_{0}^{C}$ are the preexponential factors for the $s \delta D_{\mathrm{gb}}$ and $D_{\mathrm{gb}}$ data, respectively, and $Q^{i}$ the associated activation energies. Here, given the dispersion of the $s \delta D_{\mathrm{gb}}$ and $D_{\mathrm{gb}}$ values, preexponential factors and activation energies could not be determined with accuracy. Nevertheless, Fig. 10 suggests that at $400-450{ }^{\circ} \mathrm{C}, \mathrm{s}$ cannot be vastly different from 1 (the vertical offset between the two data series is not large). Values of $s \delta D_{\text {gb }}$ from the literature have been added to the graph; an Arrhenius relationship fitted to the Čermák data [13] is plotted as a dashed line. The extrapolation of this line crosses the present $\mathrm{C}$ regime data, within the experimental dispersion. This further indicates that in the temperature range investigated here, $s$ could not be vastly different from 1 .

Another observation provides information about $s$. Two diffusion experiments were conducted at 470 and $515{ }^{\circ} \mathrm{C}$ with $\alpha / s$ values of 0.3 and 0.4 , that is, in conditions between the $\mathrm{B}$ and $\mathrm{C}$ regime if $s=1$. The profiles are plotted in $(z, \ln I)$ coordinates in Fig. 7(b), where after a smooth transition, the tails are seen to be linear. Plots in $\left(z, \operatorname{erfc}^{-1}(k \cdot I)\right)$ coordinates (not shown here) kept a visible curvature over larger depths, suggesting that these experimental conditions did not fall in the $\mathrm{C}$ regime. It follows that at $470-515{ }^{\circ} \mathrm{C}, s$ cannot be much larger than 1 . 
Finally, Fig. 10 indicates that the Arrhenius parameters obtained from the $s \delta D_{\mathrm{gb}}$ data measured by Čermák for $\mathrm{Cr}$ diffusion in pure $\mathrm{Ni}$ at $585-935{ }^{\circ} \mathrm{C}$ [13], $P_{0}=$ $1 \times 10^{-6} \mathrm{~cm}^{3} / \mathrm{s}$ and $Q=190 \mathrm{~kJ} / \mathrm{mol}$, can be used to calculate $s \delta D_{\mathrm{gb}}$ down to $350{ }^{\circ} \mathrm{C}$. It should be emphasized that a value calculated this way represents an average over many grain boundaries, and that variations of 2 orders of magnitude around this average can occur when considering individual grain boundaries.

\subsection{Shape of the intensity-depth profiles}

A remarkable feature of the present results is the variability observed in the shape of the intensity-depth profiles - and the proportion of profiles that did not take the shape expected from the analytical solutions of the grain boundary diffusion problem. The main peculiarities are discussed now.

A flat diffusion tail could in principle reflect equilibrium grain boundary segregation of $\mathrm{Cr}$ impurities originally present in the $\mathrm{Ni}$ substrate. However, some of the flat profiles observed here had levels over 30 times above the background level (see Fig. 6 for example); given the ratio between grain boundary width $(0.5 \mathrm{~nm})$ and the analyzed length (33 $\mathrm{\mu m}$ for annealed specimens), this would have required a segregation factor in excess of $10^{6}$, which, as shown above, is unlikely in the $\mathrm{Cr} / \mathrm{Ni}$ system.

Alternatively, a diffusion tail may appear flat but in fact have a small negative slope, due to a very large grain boundary diffusion coefficient. Tails in the present work generally had small slopes due to the long diffusion times, relative to the large $D_{\text {gb }} / D_{\mathrm{v}}$ ratio in this system. Some of the profiles recorded at $563{ }^{\circ} \mathrm{C}$ (Fig. 6), for example, had slopes close to the threshold value of $10^{-4} \mathrm{~nm}^{-1}$. The grain boundary diffusivity would just have to be a few times higher to produce an apparently flat profile - a possible scenario, given the dispersion of $D_{\mathrm{gb}}$ values (Fig. 10).

Another feature should be considered here: the fact that some profiles had several linear parts, with distinct slopes, suggests that regions of varying diffusivities were traversed during sputtering. In the annealed substrates, with equiaxed grains about $1 \mathrm{~mm}$ large, the probability of a $\mu \mathrm{m}$-deep profile starting in a given grain boundary and crossing a triple junction into another grain boundary is very low; instead, regions of varying diffusivities must have existed within a given boundary, likely because of local fluctuations of the grain boundary plane orientation. Furthermore, diffusivities were found to vary widely at a given temperature. The combination of these two features may generate situations in which the boundary conditions of the diffusion problem are different from one grain boundary region to another. For instance, if the diffusivity is large in region 1 , and much lower in region 2 , the tracer concentration at the region 1 /region 2 junction is expected to rise until it is close to the volume concentration, as the tracer flux in region 1 decreases to equilibrate with the flux in region 2. This would have the effect of producing a near-flat profile in region 1, as illustrated in Fig. 11.

Another remarkable aspect of the experimental results is the occurrence of diffusion tails with positive slopes, suggesting that the $\mathrm{Cr}$ flux is locally reversed, i.e., that $\mathrm{Cr}$ diffuses toward the surface. This could occur in a situation where, again, two adjoining regions have widely different grain boundary diffusivities, but the first region has the lower diffusivity (reverse of Fig. 11). If region 2 is connected to the surface via high diffusivity boundaries, $\mathrm{Cr}$ could accumulate at the region 1 /region 


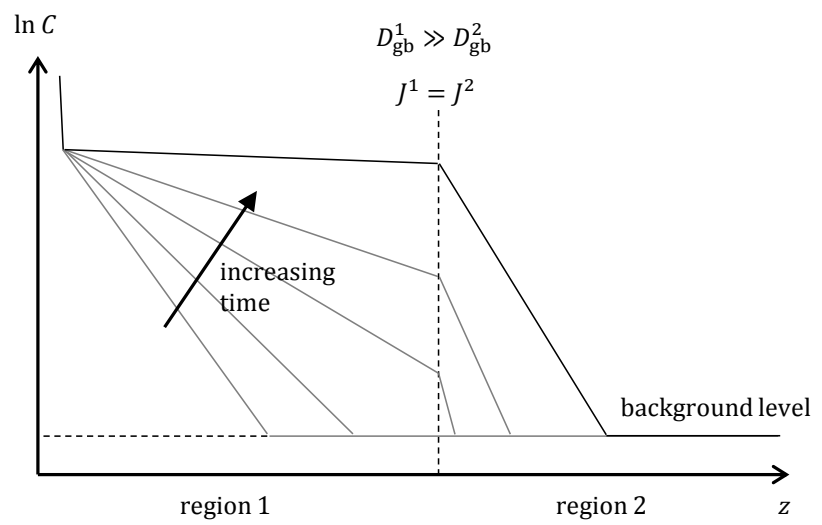

Figure 11: Scheme of concentrations in two adjoining regions of widely different grain boundary diffusivities, leading to a nearly flat concentration-depth profile in the first region (see text).

2 interface and cause a reverse flux in region 1 [25]. Alternatively, a positive slope can be seen as indicating Cr diffusion up its concentration gradient. Uphill diffusion can emerge from interactions between diffusing species, including point defects. Vacancy-solute interactions are known to cause segregation in irradiated alloys via a drag effect [32], and dealloying in nanoparticles via the inverse Kirkendall effect [33]. How vacancies would interact with $\mathrm{Cr}$ in the present case, where $\mathrm{Cr}$ diffuses from the surface via grain boundaries into a dilute solid-solution, is not known at present; this will require further investigation.

Although their origins remain to be elucidated, it is believed that the positive slopes reflect diffusion processes and are not due to SIMS measurement artefacts. Indeed, the profiles reported here were obtained with the same experimental procedure than was used to measure volume diffusion coefficients [17], which were found to be in good agreement with literature values obtained by conventional sectioning/grinding techniques.

Diffusion in individual grain boundaries has been studied in the past using bicrystals and conventional sectioning/grinding (see examples in Refs. [1,8,9], for instance). However, local measurements of diffusion in individual grain boundaries of a general polycrystal have not been reported before, to our knowledge. The present results indicate that a significant proportion of grain boundaries in polycrystalline $\mathrm{Ni}$ are subject to some sort of abnormal diffusion. Further work should be devoted to the relationships between grain boundary structure, segregation behavior and diffusivity. Useful information could be obtained by combining local depth profiling by SIMS with local chemical and structural analysis, via specimen lift-out by focused-ion beam milling, followed by atom probe tomography or transmission electron microscopy.

\subsection{Use of cold-rolled substrates}

The discussion last turns to the case of the cold-rolled substrates. The recent interest for ultrafine-grained materials has encouraged diffusion work in substrates prepared by severe plastic deformation. In particular, studies of Ni-self diffusion using speci- 
mens made by equal channel angular pressing [34] or high pressure torsion [35] have explored the relations between the type and mobility of boundaries and the associated diffusivity. These materials displayed ultrafast diffusivity, which was associated with the motion of the recrystallization front. In contrast, no ultrafast diffusivity was observed when diffusion took place in a cold-rolled substrate after partial annealing [36]. This was attributed in Ref. [36] to the lack of a mobile recrystallization front during the diffusion treatment, and to the lack of far-from-equilibrium grain boundary states otherwise associated with ultrafast diffusivity.

The present experiments on cold-rolled Ni substrates were done in conditions similar to Ref. [36]: despite the absence of a pre-diffusion annealing treatment in the present study, diffusion essentially took place in the recrystallized state due to the fast recrystallization at the temperatures of interest. Specifically, the straight diffusion tails observed at large depths reflected diffusion through a relatively stable microstructure. The smooth transition from the direct volume diffusion part into the diffusion tail (Fig. 7), however, is a trace of fast diffusion through the rapidly evolving microstructure in the very beginning of the diffusion heat treatments, during recrystallization (in contrast with the sharp transitions observed with pre-annealed substrates, Fig. 5).

A quantitative study of this fast diffusion would require performing experiments at temperatures where recrystallization is slower (where its time scale is comparable to that of the diffusion anneal). Attempts at doing so at $300-400{ }^{\circ} \mathrm{C}$ were unsuccessful, in part due to the small amount of tracer diffusing at these temperatures, relative to the substrate impurity level. Apart from using substrates of higher purity (which would recrystallize even faster), another avenue worth pursuing would be to alloy $\mathrm{Ni}$ with elements that slow its recrystallization, without interfering with the $\mathrm{Cr}$ signal. In these conditions, analyzing the shape of profiles recorded by SIMS, as was done here, would provide useful information and complement the results obtained from radiotracer studies.

\section{Conclusions}

Chromium diffusion in individual grain boundaries from annealed $\mathrm{Ni}$ polycrystals produced intensity-depth profiles with a variety of shapes. This included features that could not be understood in terms of ordinary solutions of the grain boundary diffusion problem. Smooth and abrupt transitions in diffusion tails were observed, which are indicative of local variations in boundary diffusivity. Unusual features also included parts of diffusion tails with zero and positive slopes. Both are believed to be caused by tracer accumulation at the interface between regions of markedly different diffusivities.

The conditions pertaining to the measurement of B-type profiles by SIMS, in particular the relatively shallow measurement depths, led us to determine $s \delta D_{\mathrm{gb}}$ values from a general solution of the grain boundary diffusion problem. The computer code and an example experimental profile are provided as Supplementary materials. The efforts to be made in processing profiles should be commensurate with the accuracy that can be reasonably pursued considering the experimental uncertainty and dispersion. Here, using the common method of a constant $A_{6 / 5}$ gradient given by Le Claire's approximation would have produced errors of about $20-40 \%$ on $s \delta D_{\mathrm{gb}}$ 
values (see the Appendix). This is not large considering the dispersion of the $s \delta D_{\mathrm{gb}}$ values. The analysis of exact and approximate solutions of the grain boundary diffusion problem carried out here extends the work of Le Claire and others [8], in particular in the domain of shallow measurement depths. This should be of interest in studies of grain boundary diffusion by SIMS and similar techniques.

The diffusion parameters obtained from $\mathrm{B}$ and $\mathrm{C}$ regime experiments numerically reflected the dispersion observed in the profiles, and the significant effect of misorientation on diffusivity. Aside from this dispersion, the present results were found to be consistent with the trend reported in the literature for $\mathrm{Cr}$ diffusion in $\mathrm{Ni}$ at higher temperatures.

The present results demonstrate the interest of using SIMS in grain boundary diffusion studies, and the diversity of grain boundary diffusivities in a polycrystal. A consequence of this dispersion is that it contributes to the apparently stochastic nature (on a macroscopic level) of some of the problems that rely on grain boundary properties, such as intergranular corrosion and cracking of a polycrystalline alloy.

\section{Declaration of interests}

None.

\section{Acknowledgments}

This project has received funding from the Euratom research and training programme 2014-2018 under Grant Agreement No 661913 (SOTERIA project). This article reflects only the author's view and the European Commission is not responsible for any use that may be made of the information it contains.

The authors would like to thank C. Guerre (CEA/DEN/SCCME) for providing the Cr powder, P. Bonnaillie and A. Morellec (CEA/DEN/SRMP) for performing the recrystallization heat treatments, F. Barcelo (CEA/DEN/SRMA) for performing the EBSD analyses, S. Bosonnet and K. Ginestar (CEA/DEN/SCCME) for their help setting up the diffusion heat treatments, and S. Divinski (University of Münster) for helpful discussions.

\section{Supplementary materials}

Supplementary material associated with this article can be found, in the online version, at https://doi.org/10.1016/j.mtla.2019.100283.

\section{Appendix Analysis of the concentration gradients}

The profiles recorded after diffusion in the B regime were processed using the general solution of the diffusion equation, Eqs. (4-7). In order to evaluate the error that would be made using Le Claire's method with a constant $A_{6 / 5}=0.78$, it is useful to consider the range covered by the parameters $\alpha, \beta$ and $\eta \beta^{-1 / 2}$ in the present study; these are represented graphically in Fig. A1.

Figure A2 shows the $A_{6 / 5}$ gradient calculated using Le Claire's approximation for $C_{2}$, Eq. (10), for $\beta$ values above $10^{2}$. The contribution of $C_{1}$ is included, and is 

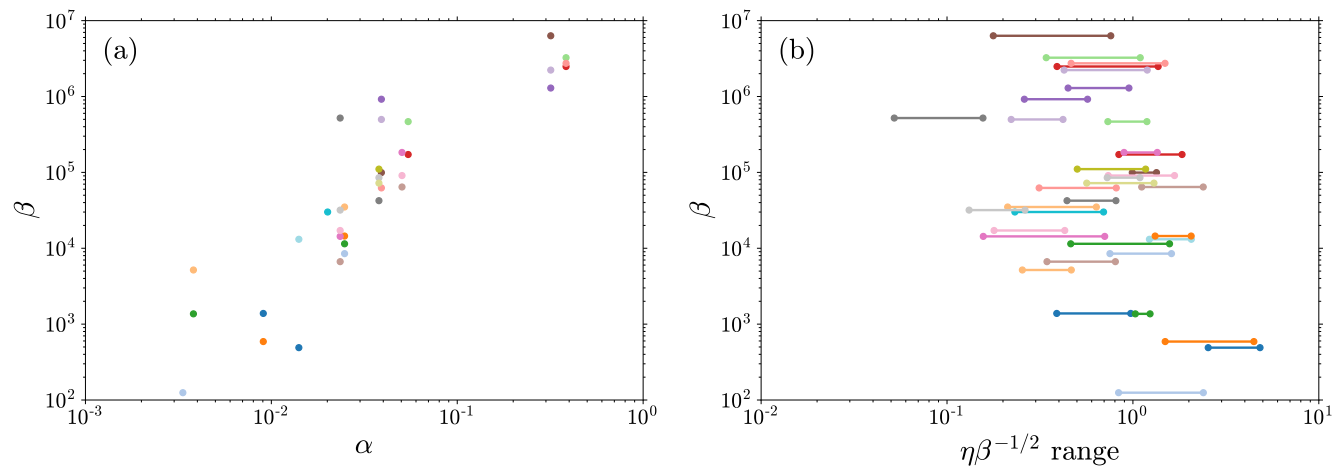

Figure A1: Ranges covered by the $\alpha, \beta$ and $\eta \beta^{-1 / 2}$ parameters in the $\mathrm{B}$ and $\mathrm{B} / \mathrm{C}$ regime experiments. See definitions in Section 4.1: $\alpha$ is a function of temperature, $\beta$ depends on the temperature and on the $s \delta D_{\mathrm{gb}}$ value measured on a given profile, and $\eta \beta^{-1 / 2}$ is a dimensionless variable for depth, also function of temperature and $s \delta D_{\mathrm{gb}}$. In (b), each $\eta \beta^{-1 / 2}$ range corresponds to the depth range over which the slope of a profile was measured.
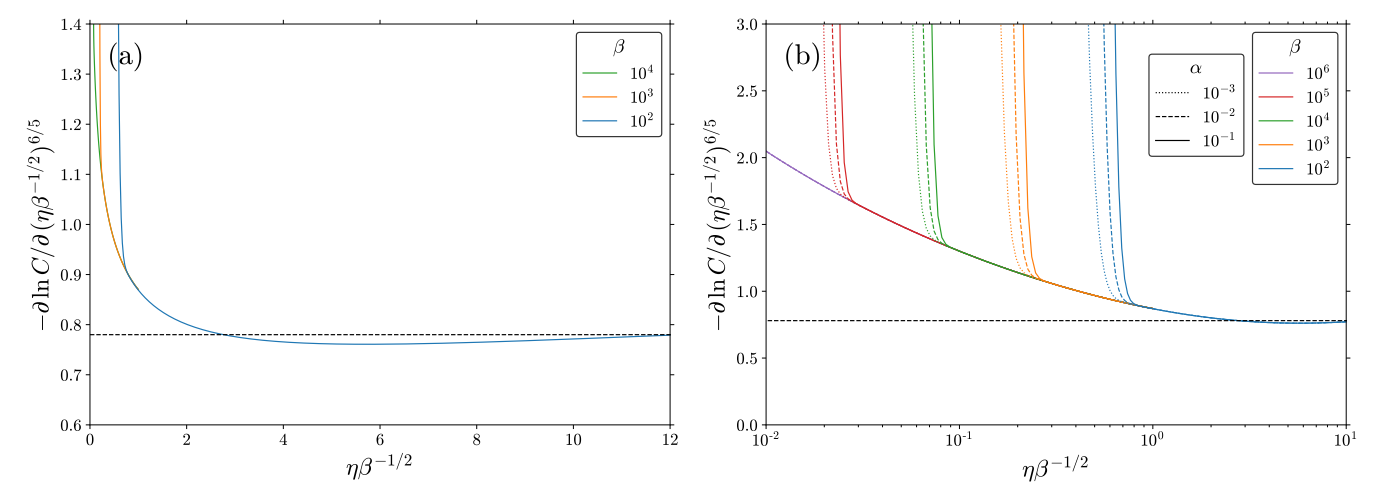

Figure A2: $A_{6 / 5}$ gradient computed from $C=C_{1}+C_{2}$, with $C_{2}$ given by Eq. (10). The $\eta \beta^{-1 / 2}$ scale is linear in (a), logarithmic in (b).

reflected in the near-vertical parts at low $\eta \beta^{-1 / 2}$ values. Le Claire's observations that $A_{6 / 5}$ is independent of $\beta$ and varies slowly with $\eta \beta^{-1 / 2}$ holds for $\eta \beta^{-1 / 2}$ greater than about 2 . In the present work, the slopes of the diffusion tails were mostly measured at depths corresponding to $\eta \beta^{-1 / 2}$ values between 0.1 and 1 . The evolution of $A_{6 / 5}$ in this range is better represented using a logarithmic scale for $\eta \beta^{-1 / 2}$, Fig. A2(b). The point at which the contribution from direct diffusion from the surface $\left(C_{1}\right)$ becomes significant depends on $\beta$ (and weakly on $\alpha$ ). Here the smaller values were obtained for large $\beta$ values (Fig. A1(b)), such that $A_{6 / 5}$ was never affected by $C_{1}-$ in practice, this corresponds to the obvious fact that the slopes were measured from the diffusion tails. More importantly, the $A_{6 / 5}$ values arising from $C_{2}$ are seen to vary significantly in the $\eta \beta^{-1 / 2}$ range of interest. These $A_{6 / 5}$ values are independent of $\beta$ or $\alpha$.

Figure A3(a) compares $A_{6 / 5}$ calculated with the approximate $C_{2}$ (Eq. (10)) and with the exact $C_{2}$ (Eq. (6)), for a given $\beta$. The two gradients start to differ signif- 

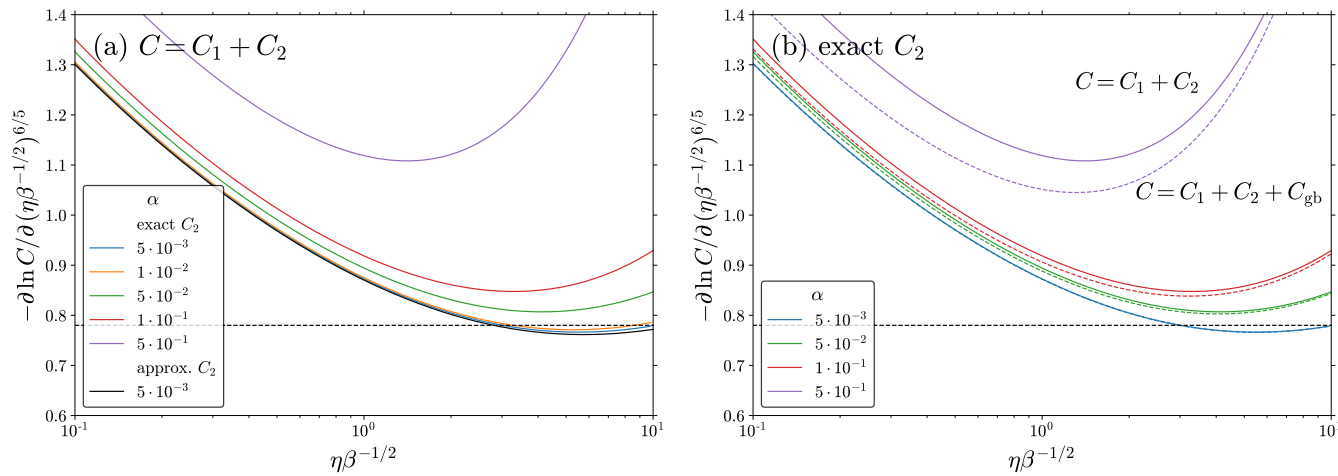

Figure A3: (a) $A_{6 / 5}$ gradient computed from $C=C_{1}+C_{2}$, using either the approximate $C_{2}$, Eq. (10), or the exact $C_{2}$, Eq. (6); (b) comparison of gradients obtained from $C=C_{1}+C_{2}$ (solid lines) and $C=C_{1}+C_{2}+C_{\mathrm{gb}}$ (dashed lines), using the exact $C_{2}$, Eq. (6). In all cases $\beta=10^{4}$.

icantly for $\alpha$ greater than $10^{-2}$, which was the case for most of the present experiments. In Fig. A3(b), the contribution from the tracer inside the grain boundary, $C_{\mathrm{gb}}$, is also included when calculating $A_{6 / 5}$. Kaur et al. [8] recommend $\alpha<5 \times 10^{-3}$ to ensure that $C_{\mathrm{gb}}$ can be neglected if concentrations are to be measured with a $0.5 \%$ accuracy, and add that $\alpha<5 \times 10^{-2}$ would be acceptable in most cases. Paul et al. [30] use a less strict criterion, $\alpha<0.1$. Relative to the other factors of interest here, we also conclude that $C_{\mathrm{gb}}$ has a significant influence only for $\alpha$ greater than 0.1 . Here, this concerned only the two experiments in between the $\mathrm{B}$ and $\mathrm{C}$ regimes (Fig. 7(b)). It is noted that $A_{6 / 5}$ is not made any less constant because of the $C_{\mathrm{gb}}$ contribution - indeed, the diffusion tails appeared reasonably linear.

According to Eq. (11), which holds regardless of the shape taken by $\mathrm{C}$, for a given diffusion tail, $s \delta D_{\mathrm{gb}}$ is proportional to $\left(A_{n}\right)^{2 / n}$. The error made by using Le Claire's method with a constant $A_{6 / 5}=0.78$ (noted $A_{6 / 5}^{\mathrm{LC}}$ ) is now evaluated by considering the ratio $\left(\frac{A_{6 / 5}^{\mathrm{LC}}}{A_{6 / 5}^{\text {exact }}}\right)^{5 / 3}$, where $A_{6 / 5}^{\text {exact }}$ is the $A_{6 / 5}$ gradient computed from the general form for $C$, Eqs. (4-7), and averaged over the $\eta \beta^{-1 / 2}$ range of interest. Figure A4 shows this ratio, where the average was done over a $\eta \beta^{-1 / 2}$ range of width 0.8 (this was the average of experimental values) and of variable midpoint, so as to cover the spread of experimental conditions. The graph shows that in the conditions of interest, a $s \delta D_{\mathrm{gb}}$ value calculated with Le Claire's method would always be underestimated, by a factor of $20-40 \%$ (and up to $50 \%$ in the particular cases where $\alpha$ is $0.3-0.4$, and the contribution of $C_{\mathrm{gb}}$ is significant).

Finally, Fig. A5 shows the $A_{n}$ gradient also calculated with all contributions, $C=C_{1}+C_{2}+C_{\mathrm{gb}}$ using Eqs. (4-7), but this time with $n=1$. Considering that the experimental $\eta \beta^{-1 / 2}$ values were mostly comprised between 0.2 and 2 (Fig. A1), the comparison of Fig. A5 and Fig. A3(b) shows that $A_{1}$ is no less constant than $A_{6 / 5}$ in the conditions of interest. This is consistent with the observation that the experimental diffusion tails were slightly more linear when plotted in $(z, \ln I)$ coordinates, compared to $\left(z^{6 / 5}, \ln I\right)$ coordinates. 


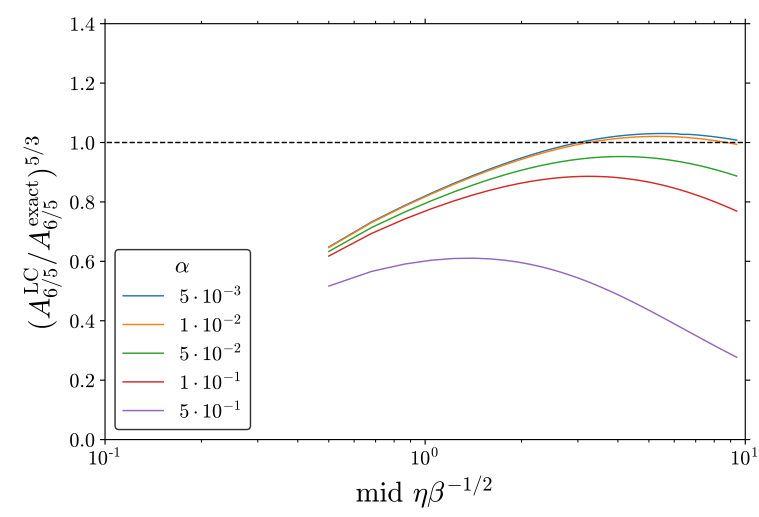

Figure A4: $\left(\frac{A_{6 / 5}^{\mathrm{LC}}}{A_{6 / 5}^{\text {exact }}}\right)^{5 / 3}$ ratio where $A_{6 / 5}^{\text {exact }}$ was computed from the general form for $C$, Eqs. (4-7), over a $\eta \beta^{-1 / 2}$ range of width 0.8 and of variable midpoint, with $\beta=10^{4}$.

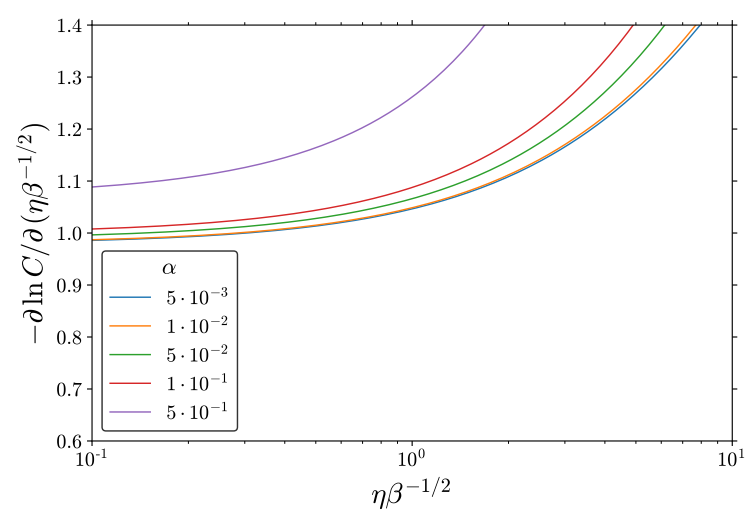

Figure A5: $A_{1}$ gradient computed using the general form for $C$, using Eqs. (4-7), with $\beta=10^{4}$.

\section{References}

[1] R. W. Balluffi, Grain boundary diffusion mechanisms in metals, Metallurgical Transactions B 13 (1982) pp. 527-553. doi: 10.1007/BF02650011.

[2] F. Delabrouille, L. Legras, F. Vaillant, P. Scott, B. Viguier and E. Andrieu, Effect of the chromium content and strain on the corrosion of nickel based alloys in primary water of pressurized water reactors, in Proceedings of the 12th International Conference on Environmental Degradation of Materials in Nuclear Power Systems-Water Reactors (T. Allen, P. King and L. Nelson, eds.) pp. 903-11 TMS (The Minerals, Metals \& Materials Society) (2005).

[3] J. Panter, B. Viguier, J.-M. Cloué, M. Foucault, P. Combrade and E. Andrieu, Influence of oxide films on primary water stress corrosion cracking initiation of alloy 600, Journal of Nuclear Materials 348 (2006) pp. 213-221. doi: 10.1016/j.jnucmat.2005.10.002. 
[4] L. E. Thomas and S. M. Bruemmer, High-resolution characterization of intergranular attack and stress corrosion cracking of alloy 600 in high-temperature primary water, Corrosion 56 (2000) pp. 572-587. doi: 10.5006/1.3280561.

[5] Y. S. Lim, H. P. Kim and S. S. Hwang, Microstructural characterization on intergranular stress corrosion cracking of alloy 600 in PWR primary water environment, Journal of Nuclear Materials 440 (2013) pp. 46-54. doi: 10.1016/j.jnucmat.2013.03.088.

[6] D. Schreiber, M. Olszta and S. Bruemmer, Grain boundary depletion and migration during selective oxidation of $\mathrm{Cr}$ in a $\mathrm{Ni}-5 \mathrm{Cr}$ binary alloy exposed to high-temperature hydrogenated water, Scripta Materialia 89 (2014) pp. 41-44. doi: 10.1016/j.scriptamat.2014.06.022.

[7] S. Bruemmer, M. Olszta, M. Toloczko and D. Schreiber, Grain boundary selective oxidation and intergranular stress corrosion crack growth of high-purity nickel binary alloys in high-temperature hydrogenated water, Corrosion Science 131 (2018) pp. 310-323. doi: 10.1016/j.corsci.2017.11.024.

[8] I. Kaur, W. Gust and Y. Mishin, Fundamentals of grain and interphase boundary diffusion. Wiley Chichester (1995).

[9] Y. Mishin, C. Herzig, J. Bernardini and W. Gust, Grain boundary diffusion: fundamentals to recent developments, International Materials Reviews 42 (1997) pp. 155-178. doi: 10.1179/imr.1997.42.4.155.

[10] D. Pruthi, M. Anand and R. Agarwala, Diffusion of chromium in Inconel600, Journal of Nuclear Materials 64 (1977) pp. 206-210. doi: 10.1016/00223115(77)90026-5.

[11] P. Moulin, A. Huntz and P. Lacombe, Influence du carbone sur la diffusion du chrome et du nickel en volume et dans les joints de grains de l'alliage $\mathrm{Ni}-$ Cr 80/20, Acta Metallurgica 27 (1979) pp. 1431-1443. doi: 10.1016/00016160(79)90165-2.

[12] P. Guiraldenq and P. Poyet, Étude comparative de la diffusion intergranulaire et de la désorption ultérieure du chrome vers la surface dans les alliages Ni75Cr16Fe9 (type Inconel 600) en présence de vapeur d'eau par l'emploi du radiotraceur 51Cr, Mémoires et Études Scientifiques de la Revue de Métallurgie 77 (1980) pp. 939-949.

[13] J. Čermák, Grain boundary self-diffusion of ${ }^{51} \mathrm{Cr}$ and ${ }^{59} \mathrm{Fe}$ in austenitic Ni-FeCr alloys, Materials Science and Engineering: A 148 (1991) pp. 279-287. doi: 10.1016/0921-5093(91)90830-G.

[14] T.-F. Chen, G. P. Tiwari, Y. Iijima and K. Yamauchi, Volume and grain boundary diffusion of chromium in Ni-base Ni-Cr-Fe alloys, Materials Transactions 44 (2003) pp. 40-46. doi: 10.2320/matertrans.44.40.

[15] B. Chetroiu, Corrosion sous contrainte de l'alliage 600 en millieu primaire des REP: étude de la diffusion du chrome. PhD thesis École Nationale Supérieure des Mines de Paris (2015). https://pastel.archives-ouvertes. fr/tel-01157822.

[16] C. Desgranges, F. Lequien, E. Aublant, M. Nastar and D. Monceau, Depletion and voids formation in the substrate during high temperature oxidation of $\mathrm{Ni}-$ 
Cr alloys, Oxidation of Metals 79 (2013) pp. 93-105. doi: 10.1007/s11085-0129328-0.

[17] T. Gheno, F. Jomard, C. Desgranges and L. Martinelli, Tracer diffusion of Cr in $\mathrm{Ni}$ and $\mathrm{Ni}-22 \mathrm{Cr}$ studied by SIMS, Materialia 3 (2018) pp. 145-152. doi: 10.1016/j.mtla.2018.08.004.

[18] L. G. Harrison, Influence of dislocations on diffusion kinetics in solids with particular reference to the alkali halides, Trans. Faraday Soc. 57 (1961) pp. 11911199. doi: 10.1039/TF9615701191.

[19] D. Gaertner, G. Wilde and S. V. Divinski, Grain boundary diffusion and segregation of ${ }^{57} \mathrm{Co}$ in high-purity copper: Radiotracer measurements in Band C-type diffusion regimes, Acta Materialia 127 (2017) pp. 407-415. doi: 10.1016/j.actamat.2017.01.045.

[20] R. Whipple, Concentration contours in grain boundary diffusion, Philosophical Magazine 45 (1954) pp. 1225-1236. doi: 10.1080/14786441208561131.

[21] Y. M. Mishin, A model of grain boundary diffusion in coarse-grained polycrystals, Physica Status Solidi (a) 133 (1992) pp. 259-267. doi: 10.1002/pssa.2211330207.

[22] A. D. Le Claire, The analysis of grain boundary diffusion measurements, British Journal of Applied Physics 14 (1963) p. 351. doi: 10.1088/0508-3443/14/6/317.

[23] J. C. Fisher, Calculation of diffusion penetration curves for surface and grain boundary diffusion, Journal of Applied Physics 22 (1951) pp. 74-77. doi: 10.1063/1.1699825.

[24] H. S. Levine and C. J. MacCallum, Grain boundary and lattice diffusion in polycrystalline bodies, Journal of Applied Physics 31 (1960) pp. 595-599. doi: $10.1063 / 1.1735634$.

[25] S.V. Divinski, private communication.

[26] E. Jones, E. Oliphant, P. Peterson and et al., Scipy: Open source scientific tools for Python, (2001). http://www.scipy.org/.

[27] D. Shaw and T. L. Shaw, A least squares method for fitting diffusion data to the Whipple/Suzuoka equations for grain boundary diffusion, Journal of Applied Physics 84 (1998) pp. 3586-3592. doi: 10.1063/1.368534.

[28] D. Gryaznov, J. Fleig and J. Maier, An improved procedure for determining grain boundary diffusion coefficients from averaged concentration profiles, Journal of Applied Physics 103 (2008) p. 063717. doi: 10.1063/1.2887993.

[29] D. Monceau and B. Pieraggi, Determination of parabolic rate constants from a local analysis of mass-gain curves, Oxidation of Metals 50 (1998) pp. 477-493. doi: 10.1023/a:1018860909826.

[30] A. Paul, T. Laurila, V. Vuorinen and S. V. Divinski, Thermodynamics, diffusion and the Kirkendall effect in solids. Springer (2014).

[31] S. V. Divinski, G. Reglitz and G. Wilde, Grain boundary self-diffusion in polycrystalline nickel of different purity levels, Acta Materialia 58 (2010) pp. 386395. doi: 10.1016/j.actamat.2009.09.015. 
[32] T. Schuler, P. Bellon, D. R. Trinkle and R. S. Averback, Modeling the longterm evolution of dilute solid solutions in the presence of vacancy fluxes, Phys. Rev. Materials 2 (2018) p. 073605. doi: 10.1103/PhysRevMaterials.2.073605.

[33] A. Gusak and K. Tu, Interaction between the Kirkendall effect and the inverse Kirkendall effect in nanoscale particles, Acta Materialia 57 (2009) pp. 33673373. doi: $10.1016 /$ j.actamat.2009.03.043.

[34] S. V. Divinski, G. Reglitz, H. Rösner, Y. Estrin and G. Wilde, Ultra-fast diffusion channels in pure $\mathrm{Ni}$ severely deformed by equal-channel angular pressing, Acta Materialia 59 (2011) pp. 1974-1985. doi: 10.1016/j.actamat.2010.11.063.

[35] D. Prokoshkina, L. Klinger, A. Moros, G. Wilde, E. Rabkin and S. V. Divinski, Effect of recrystallization on diffusion in ultrafine-grained Ni, Acta Materialia 69 (2014) pp. 314-325. doi: 10.1016/j.actamat.2014.02.002.

[36] M. Shepelenko, L. Klinger, E. Rabkin, A. Berner, D. Prokoshkina, G. Reglitz, J. Fiebig, G. Wilde and S. V. Divinski, Recovery, recrystallization and diffusion in cold-rolled Ni, International Journal of Materials Research 106 (2015) pp. 554-564. doi: 10.3139/146.111217. 